\title{
Langevin Equation for the Rayleigh model with finite-ranged interactions
}

\author{
Alexander V. Plyukhin and Jeremy Schofield, \\ Chemical Physics Theory Group, Department of Chemistry, \\ University of Toronto, Toronto, Ontario, Canada M5S $3 \mathrm{H} 6$
}

(October 29, 2018)

\begin{abstract}
Both linear and nonlinear Langevin equations are derived directly from the Liouville equation for an exactly solvable model consisting of a Brownian particle of mass $M$ interacting with ideal gas molecules of mass $m$ via a quadratic repulsive potential. Explicit microscopic expressions for all kinetic coefficients appearing in these equations are presented. It is shown that the range of applicability of the Langevin equation, as well as statistical properties of random force, may depend not only on the mass ratio $m / M$ but also by the parameter $N m / M$, involving the average number $N$ of molecules in the interaction zone around the particle. For the case of a short-ranged potential, when $N \ll 1$, analysis of the Langevin equations yields previously obtained results for a hardwall potential in which only binary collisions are considered. For the finite-ranged potential, when multiple collisions are important $(N \gg 1)$, the model describes nontrivial dynamics on time scales that are on the order of the collision time, a regime that is usually beyond the scope of more phenomenological models.
\end{abstract}

\section{INTRODUCTION}

The explicit derivation from first principles of the Langevin equation, describing the evolution of a small number of variables in a complex system, is often necessary since in many cases the statistical properties of the random force, the range of applicability and even the form of the equation are far from evident [1]. The need for microscopic considerations is especially compelling in the case of a nonlinear Langevin equation where the usual phenomenological approach of adding stochastic terms to the deterministic nonlinear equation describing the relaxation of targeted variables may be inadequate [2]. Although statistical properties of the random force in the nonlinear Langevin equation may be deduced phenomenologically in some cases [3], it is generally necessary to start from the microscopic behavior of the system in order to construct the appropriate form of the equation.

The conventional systematic method for deriving the Langevin equation (LE) for a Brownian particle exploits Mori's projection-operator techniques [1], which allow the transformation of the microscopic Liouville equation to a non-Markovian predecessor of the LE, generally known as the generalized Langevin equation. The LE can be then obtained by a subsequent perturbation expansion of the memory kernel appearing in the generalized Langevin equation using the square root ratio of the mass of a bath particle to that of the Brownian particle, $(m / M)^{1 / 2}$, as a perturbation parameter $\lambda$. While the first step in the derivation of the LE, involving rearrangement of the Liouville equation with projection operator methods, is an exact algebraic procedure, the question of convergence of the $\lambda$ expansion in the second step is subtle and can be strictly justified only under assumptions which are difficult to prove in general [4]. Mazur and Oppenheim developed an alternative projection-operator approach more suitable to analyse the convergence of the $\lambda$ expansion [5]. The validity of the perturbation analysis in both the Mori and the Mazur-Oppenheim approaches has been examined for an exactly solvable model consisting of tagged particle motion in a harmonic lattice [6]. Unfortunately, the convergence properties of the $\lambda$ series are trivial for this model, since all terms in the $\lambda$ expansion higher than zeroth order vanish. As a result, the memory kernel for the linear damping force in the LE does not depend on the mass ratio, and all non-linear terms are identically zero [7].

One goal of this paper is to present and analyze a simplified model that serves as a useful and non-trivial testing ground for examining some of the subtle aspects of the theory of Brownian motion. The model considered here is a generalization of the well-known Rayleigh model of a Brownian particle of mass $M$ constrained to move in one dimension and subjected to collisions with an equilibrium ideal (non-interactive) gas of particles of mass $m$. The Rayleigh model is perhaps the oldest model of non-equilibrium statistical physics [8], and has attracted much attention over the years, with early work [9-11] focusing on the model as a test of the systematic derivation of macroscopic kinetic equations for the heavy particle from the master equation. More recent investigations have examined the stationary and transient solutions of the asymmetric Rayleigh model in which the thermodynamic parameters characterizing the gas to the left and right of the piston differ [12]. In all these studies, the interaction between the Brownian particle and the bath is assumed to be short-ranged with a negligible collision time $\tau_{c}$. Only binary collisions are considered in this model because the range of interaction is assumed to be short compared to the average distant between bath particles. The usual starting point for analysis of the Rayleigh model with binary collisions is a Markovian master equation for particle's velocity distribution function. The master equation is only an approximate form of the fully 
microscopic Liouville equation, and valid only for time scales longer than $\tau_{c}$. To test many aspects of the theory of Brownian motion, it is essential to start from a fully microscopic description of the dynamics directly from the Liouville equation so that any non-Markovian character of bath correlations is properly incorporated and particle dynamics on time scales less than $\tau_{c}$ can be described. To this end, one may generalize the interaction between the bath and Brownian particles from a hard-wall to a parabolic repulsive potential. The generalized model is analytically solvable, allowing explicit calculation of all terms appearing in the derivation of both the linear and nonlinear LE beyond the binary collision approximation. We demonstrate that in addition to the small parameter $\lambda=(\mathrm{m} / M)^{1 / 2}$, the character of the dynamics of a tagged particle can be governed by an additional parameter, $N^{1 / 2} \lambda$, involving the average number $N$ of bath particles simultaneously interacting with the Brownian particle. For a large Brownian particle, it is shown that when $N \gg 1$ and multiple collisions are important, the parameter of the formal $\lambda$-expansion is actually $N^{1 / 2} \lambda$.

The paper is organized as follows: In section 2, the Mazur-Oppenheim approach is reviewed to provide groundwork for all subsequent analysis. In section 3, the structure of terms in the $\lambda$ expansion is examined and presented in a convenient form. In section 4, the general formalism is applied to the Rayleigh model with a quadratic repulsive potential describing bath-Brownian particle interactions, and the LE is derived for the heavy particle. The nonlinear LE is obtained in section 5 and various aspects of this equation are discussed. Finally, a few concluding remarks are made in section 6 .

\section{BASIC EQUATIONS}

The Hamiltonian for a Brownian system composed of a tagged particle of mass $M$ in a bath of point particles of mass $m$ is

$$
\begin{aligned}
H & =\frac{P^{2}}{2 M}+H_{0}, \\
H_{0} & =\sum_{i} \frac{p_{i}^{2}}{2 m}+U(x, X),
\end{aligned}
$$

where $x=\left\{x_{i}\right\}$ and $p_{i}$ are positions and momenta of bath particles, $X$ and $P$ are those of the Brownian (or tagged) particle, and $H_{0}$ is the Hamiltonian of the bath in the field of the tagged particle fixed at $X$. One can expect that on average $P \sim \sqrt{M k_{B} T}$, where $k_{B}$ is Boltzmann's constant and $T$ is the temperature, and that the scaled momentum $P_{*}=\lambda P$, where $\lambda=\sqrt{m / M}$, is of the same order as the average momentum of a bath particle. In terms of scaled momentum, one can write the Liouville operator as

$$
\begin{aligned}
\mathcal{L} & =\mathcal{L}_{0}+\lambda \mathcal{L}_{1} \\
\mathcal{L}_{0} & =\sum_{i}\left\{\frac{p_{i}}{m} \frac{\partial}{\partial x_{i}}+F_{i} \frac{\partial}{\partial p_{i}}\right\} \\
\mathcal{L}_{1} & =\frac{P_{*}}{m} \frac{\partial}{\partial X}+F \frac{\partial}{\partial P_{*}}
\end{aligned}
$$

where $F_{i}=-\nabla_{x_{i}} U$ and $F=-\nabla_{X} U$ are the forces on the $i$-th bath particle and on the Brownian particle, respectively. The operator $\mathcal{L}_{0}$ dictates the dynamics of the bath in the field of the fixed Brownian particle.

If the mass of the tagged particle is large (i.e. a Brownian particle), one might intuitively expect that inertial effects of the particle's motion are small and that the force on the particle, $F(t)=e^{\mathcal{L} t} F$, is close to the pressure force, i.e. to the force on the fixed tagged particle,

$$
F_{0}(t) \equiv e^{\mathcal{L}_{0} t} F
$$

In the Mazur-Oppenheim approach [5], the force $F(t)$ is decomposed using the projection operator $\mathcal{P}$ which averages a dynamical variable $A$ over the canonical distribution $\rho=Z^{-1} \exp \left(-\beta H_{0}\right)$, for bath variables at fixed position of the tagged particle,

$$
\mathcal{P} A=\langle A\rangle \equiv \int \rho A \prod_{i} d x_{i} d p_{i}
$$

where $Z$ is the canonical partition function and $\beta=1 / k_{B} T$. Using the operator identity [13]

$$
e^{(\mathcal{A}+\mathcal{B}) t}=e^{\mathcal{A} t}+\int_{0}^{t} d \tau e^{\mathcal{A}(t-\tau)} \mathcal{B} e^{(\mathcal{A}+\mathcal{B}) \tau},
$$


with $\mathcal{A}=\mathcal{L}$ and $\mathcal{B}=-\mathcal{P} \mathcal{L}$, one may formally decompose the force $F(t)$ on the tagged particle into a "random" part and a remainder as

$$
F(t)=F^{\dagger}(t)+\int_{0}^{t} d \tau e^{\mathcal{L}(t-\tau)} \mathcal{P} \mathcal{L} F^{\dagger}(\tau)
$$

where $F^{\dagger}(t)=e^{\mathcal{Q L} t} F$ and $\mathcal{Q}=1-\mathcal{P}$. The factor $\mathcal{P} \mathcal{L} F^{\dagger}(\tau)$ in the integral in Eq. (9) can be simplified taking into account the orthogonality of $\mathcal{P}$ and $\mathcal{L}_{0}$ (i.e. $\mathcal{P} \mathcal{L}_{0}=0$ ), and the equality

$$
\left\langle\nabla_{X} F^{\dagger}(t)\right\rangle=-\beta\left\langle F_{0} F^{\dagger}(t)\right\rangle,
$$

which can be derived by integration by parts. As a result, one obtains the following exact equation of motion for the scaled momentum of the tagged particle,

$$
\frac{d P_{*}(t)}{d t}=\lambda F^{\dagger}(t)+\lambda^{2} \int_{0}^{t} d \tau e^{\mathcal{L}(t-\tau)}\left(\nabla_{P_{*}}-\frac{\beta}{m} P_{*}\right)\left\langle F F^{\dagger}(\tau)\right\rangle
$$

where $F^{\dagger}(t)$ is a zero-centered random force obeying $\left\langle F^{\dagger}(t)\right\rangle=\mathcal{P} e^{\mathcal{Q L} t} F=0$.

The random force $F^{\dagger}(t)=e^{\left(\mathcal{L}_{0}+\lambda \mathcal{Q} \mathcal{L}_{1}\right) t} F$ can be further expanded in terms of the mass ratio parameter $\lambda$ using identity (8) to obtain

$$
\begin{aligned}
F^{\dagger}(t)= & F_{0}(t)+\lambda \int_{0}^{t} d t_{1} e^{\mathcal{L}_{0}\left(t-t_{1}\right)} \mathcal{Q} \mathcal{L}_{1} F_{0}\left(t_{1}\right)+ \\
& \lambda^{2} \int_{0}^{t} d t_{1} \int_{0}^{t_{1}} d t_{2} e^{\mathcal{L}_{0}\left(t-t_{1}\right)} \mathcal{Q} \mathcal{L}_{1} e^{\mathcal{L}_{0}\left(t_{1}-t_{2}\right)} \mathcal{Q L} \mathcal{L}_{1} F_{0}\left(t_{2}\right)+\cdots
\end{aligned}
$$

Similarly, the kernel $K(t)=\left\langle F F^{\dagger}(t)\right\rangle$ appearing in the exact equation of motion (11) of the tagged particle may be expanded in a power series in $\lambda$

$$
\begin{aligned}
K(t) & =\left\langle F F^{\dagger}(t)\right\rangle=\sum_{l} K_{l}(t), \\
K_{0}(t) & =\lambda^{0}\left\langle F F_{0}(t)\right\rangle \\
K_{1}(t) & =\lambda^{1} \int_{0}^{t} d t_{1} C_{1}\left(t, t_{1}\right), \\
K_{2}(t) & =\lambda^{2} \int_{0}^{t} d t_{1} \int_{0}^{t_{1}} d t_{2} C_{2}\left(t, t_{1}, t_{2}\right) \cdots
\end{aligned}
$$

where the correlation functions $C_{l}$ are defined to be

$$
\begin{aligned}
C_{1}\left(t_{0}, t_{1}\right) & =\left\langle F\left(e^{\mathcal{L}_{0}\left(t_{0}-t_{1}\right)} \mathcal{Q} \mathcal{L}_{1}\right) F_{0}\left(t_{1}\right)\right\rangle \\
C_{2}\left(t_{0}, t_{1}, t_{2}\right) & =\left\langle F\left(e^{\mathcal{L}_{0}\left(t_{0}-t_{1}\right)} \mathcal{Q} \mathcal{L}_{1}\right)\left(e^{\mathcal{L}_{0}\left(t_{1}-t_{2}\right)} \mathcal{Q} \mathcal{L}_{1}\right) F_{0}\left(t_{2}\right)\right\rangle, \\
C_{l}\left(t_{0}, t_{1}, \cdots, t_{l}\right) & =\left\langle F\left(\prod_{i=1}^{l} e^{\mathcal{L}_{0}\left(t_{i-1}-t_{i}\right)} \mathcal{Q} \mathcal{L}_{1}\right) F_{0}\left(t_{l}\right)\right\rangle .
\end{aligned}
$$

The truncation of the $\lambda$-expansion to zeroth order, $K(t) \approx K_{0}(t)$, leads from Eq.(11) directly to the generalized Langevin equation

$$
\frac{d P_{*}(t)}{d t}=\lambda F^{\dagger}(t)-\lambda^{2} \int_{0}^{t} d \tau M_{0}(\tau) P_{*}(t-\tau)
$$

where

$$
M_{0}(t)=\frac{\beta}{m} K_{0}(t)=\frac{\beta}{M}\left\langle F F_{0}(t)\right\rangle .
$$

This approximation is sensible provided the correlation functions $C_{l}$ appearing at higher order in the $\lambda$-expansion decay on a similar $\lambda$-independent time scale $\tau_{c}$ characteristic of motions of the fixed-particle system (i.e. governed 
by $\mathcal{L}_{0}$ ). Mazur and Oppenheim [5] succeeded in proving that this is the case assuming the factorization properties $\left\langle A\left(t_{1}\right) e^{\mathcal{L}_{0} t} B\left(t_{2}\right)\right\rangle \stackrel{t>\tau_{c}}{\longrightarrow}\left\langle A\left(t_{1}\right)\right\rangle\left\langle B\left(t_{2}\right)\right\rangle$.

While being formally non-local in time, Eq. (15) can actually be written in a form that is local in time by expanding $P_{*}(t-\tau)$ around $\tau=0$ to obtain

$$
\frac{d P_{*}(t)}{d t}=\lambda F^{\dagger}(t)-\lambda^{2} \gamma_{0}(t) P_{*}(t)
$$

where $\gamma_{0}(t)=\int_{0}^{t} d \tau M_{0}(\tau)$. The non-local correction terms to this approximation are of the form [14]

$$
\lambda^{2} \int_{0}^{t} d \tau M_{0}(\tau) \int_{t-\tau}^{t} d \tau^{\prime} \dot{P}_{*}\left(\tau^{\prime}\right) \sim \lambda^{3}
$$

and are therefore of higher order in $\lambda$. Naturally, this analysis is pertinent only if the characteristic time $\tau_{c}$ for decay of $M_{0}(t)$ does not depend on $\lambda$.

The local equation (17) is applicable on arbitrary time scales and for $t \gg \tau_{c}$ assumes the form of the conventional LE with a time-independent damping coefficient $\gamma_{0}=\int_{0}^{\infty} d t M_{0}(t)$. It is then evident from (17) that the autocorrelation function of the momentum of a heavy particle decays on a time-scale $\tau_{p} \sim \lambda^{-2}$ that is much longer than the characteristic time of the bath $\tau_{c} \sim \lambda^{0}$. One can therefore expect the local form of the LE with time-independent damping to be a good approximation for Eq. (17) except at short times determined by $t<\tau_{c}$.

It will be shown below that for homogeneous bath $K_{1}(t)=0$, so the next approximation for the $\lambda$-expansion (13) is of the form $K(t) \approx K_{0}(t)+K_{2}(t)$. The equation of motion for the momentum of the tagged particle in this case includes a non-linear damping term of third order in $P_{*}$. This equation will be considered in section 5 .

\section{STRUCTURE OF THE TERMS IN $\lambda$-EXPANSION}

To examine the convergence properties of the $\lambda$-expansion and other features of the projection operator derivation of the LE, the structure of the correlation functions $C_{l}$ defined in (14) which appear in the expansion of the memory function must be analyzed. Although only the functions $C_{0}$ and $C_{2}$ are needed to obtain the nonlinear LE to lowest order in $\lambda$, it is useful to know general properties of $C_{l}$.

By inspection of the symmetry properties of the system, it is immediately apparent that the correlation functions $C_{2 n+1}$ corresponding to odd powers of $\lambda$ contain an odd number of factors $F$ and $\nabla_{X}$, and therefore vanish for isotropic systems. In fact, in the absence of external field, the dependence on the particle coordinate appears only through the difference $x_{i}-X$, and it is useful to introduce new variables $q_{i}=x_{i}-X$. Since the vectors $F=-\nabla_{X} U=\sum_{i} \nabla_{q_{i}} U$ and $\nabla_{X}=-\sum_{i} \nabla_{q_{i}}$ have negative parity and the Hamiltonian $H_{0}$ is invariant with respect to transformation $\left\{q_{i} \rightarrow-q_{i}, p_{i} \rightarrow-p_{i}\right\}$, the correlation functions $C_{2 n+1}\left(t, t_{1}, \ldots t_{2 n+1}\right)$ vanish.

Correlation functions of even orders $C_{2 n}$ do not vanish and have a rather complicated structure. For notational simplicity, we restrict the analysis to the case of one-dimensional diffusion, expecting no physical features in higher dimensions. For future development, it is convenient to define

$$
\begin{aligned}
G_{0}(t) & =F_{0}(t), \\
G_{1}\left(t, t_{1}\right) & =S\left(t-t_{1}\right) F_{0}\left(t_{1}\right), \\
G_{2}\left(t, t_{1}, t_{2}\right) & =S\left(t-t_{1}\right) S\left(t_{1}-t_{2}\right) F_{0}\left(t_{2}\right), \\
G_{s}\left(t, t_{1}, \cdots, t_{s}\right) & =S\left(t-t_{1}\right) \cdots S\left(t_{s-1}-t_{s}\right) F_{0}\left(t_{s}\right),
\end{aligned}
$$

where

$$
S\left(t_{i}-t_{l}\right)=e^{\mathcal{L}_{0}\left(t_{i}-t_{l}\right)} \frac{\partial}{\partial X}
$$

Note the property

$$
\left\langle G_{i_{1}} G_{i_{2}} \cdots G_{i_{l}}\right\rangle=0
$$

which holds for arbitrary time arguments when $i_{1}+i_{2}+\cdots+i_{l}$ and $l$ have different parities. For example, $\left\langle G_{i}\right\rangle$ and $\left\langle G_{0} G_{0} G_{i}\right\rangle$ vanish for even $i$, while $\left\langle G_{0} G_{i}\right\rangle$ and $\left\langle G_{0} G_{0} G_{0} G_{i}\right\rangle$ are zero for odd $i$.

Using the definitions above and according to Eqs. (14), the second order correlation function $C_{2}$ can be written as 


$$
\begin{aligned}
C_{2}\left(t, t_{1}, t_{2}\right)= & \left(\frac{P_{*}}{m}\right)^{2}\left\langle G_{0} G_{2}\left(t, t_{1}, t_{2}\right)\right\rangle \\
& +\frac{1}{m}\left\langle G_{0} G_{0}\left(t-t_{1}\right) G_{1}\left(t, t_{2}\right)\right\rangle \\
& -\frac{1}{m}\left\langle G_{0} G_{0}\left(t-t_{1}\right)\right\rangle\left\langle G_{1}\left(t, t_{2}\right)\right\rangle .
\end{aligned}
$$

Using cumulants, denoted by $\langle\langle\cdots\rangle$ and defined via the relations,

$$
\begin{aligned}
\langle A\rangle= & \left\langle\left\langle A_{1}\right\rangle\right\rangle, \\
\left\langle A_{1} A_{2}\right\rangle= & \left\langle A_{1}\right\rangle\left\langle A_{2}\right\rangle+\left\langle\left\langle A_{1} A_{2}\right\rangle\right\rangle, \\
\left\langle A_{1} A_{2} A_{3}\right\rangle= & \left\langle A_{1}\right\rangle\left\langle A_{2}\right\rangle\left\langle A_{3}\right\rangle+ \\
& \left\langle A_{1}\right\rangle\left\langle\left\langle A_{2} A_{3}\right\rangle\right\rangle+\left\langle A_{2}\right\rangle\left\langle\left\langle A_{3} A_{1}\right\rangle\right\rangle+\left\langle A_{3}\right\rangle\left\langle\left\langle A_{2} A_{1}\right\rangle\right\rangle+ \\
& \left\langle\left\langle A_{1} A_{2} A_{3}\right\rangle\right\rangle, \\
\vdots &
\end{aligned}
$$

the function $C_{2}$ can be written as

$$
\begin{aligned}
C_{2}\left(t, t_{1}, t_{2}\right)= & \left(\frac{P_{*}}{m}\right)^{2}\left\langle\left\langle G_{0} G_{2}\left(t, t_{1}, t_{2}\right)\right\rangle\right\rangle+ \\
& \frac{1}{m}\left\langle\left\langle G_{0} G_{0}\left(t-t_{1}\right) G_{1}\left(t, t_{2}\right)\right\rangle\right\rangle .
\end{aligned}
$$

Note that the zeroth order kernel $K_{0}$ is also a cumulant, $K_{0}=\left\langle F F_{0}(t)\right\rangle=\left\langle\left\langle G_{0} G_{0}(t)\right\rangle\right\rangle$. The relevance of cumulant representation for $C_{l}$ follows from the fact that one expects the cumulants to have similar scaling properties with respect to parameters of the system independent of their order. For example, it will be established in the next section that cumulants $\left\langle\left\langle G_{i_{1}} G_{i_{2}} \cdots G_{i_{l}}\right\rangle\right\rangle$ of any order are linear functions of the average number $N$ of particles in the interaction zone around the particle for the Rayleigh model. Therefore, for this model, the first two non-vanishing terms in the $\lambda$-expansion (13), namely $K_{0}$ and $K_{2}$, are both linear in $N$.

Consider the correlation functions $C_{2 l}$ with $l \geq 1$. It is tedious, though not difficult to establish that these correlation functions are of order $l$ in cumulants $\left\langle\left\langle G_{i_{1}} G_{i_{2}} \cdots G_{i_{l}}\right\rangle\right\rangle$. Note that $C_{2 l}$ has the contributions which are of order $l+2$ or less in $G_{i}$. First consider the terms of maximal order $l+2$ : One type of these terms are the correlation functions of the form

$$
\left\langle G_{0} G_{0} G_{i_{1}} G_{i_{2}} \cdots G_{i_{l}}\right\rangle,
$$

where here and below time arguments have been omitted for brevity. The indices $i_{1}, \cdots, i_{l}$ may take any values from the set $\{0,1, \cdots, l\}$ provided

$$
i_{1}+i_{2}+\cdots+i_{l}=l .
$$

For example, for $l=1$ the correlation function of maximal order in $G_{i}$ which contributes to $C_{2}$ is $\left\langle G_{0} G_{0} G_{1}\right\rangle$, as can be seen from Eq. (23). For $l=2$ the function $C_{4}$ includes contributions of the form $\left\langle G_{0} G_{0} G_{0} G_{2}\right\rangle$ and $\left\langle G_{0} G_{0} G_{1} G_{1}\right\rangle$, and so on.

All other contributions of order $l+2$ in $G_{i}$ can be written as products of correlation functions of lower orders which can be obtained dividing the sequences

$$
G_{0}\left(\tau_{1}\right) G_{0}\left(\tau_{2}\right) G_{i_{1}}\left(\tau_{3}\right) G_{i_{2}}\left(\tau_{4}\right) \cdots G_{i_{l}}\left(\tau_{l+2}\right)
$$

into groups in all possible ways without permuting functions. For example, for $l=2$ the three remaining terms are

$$
\begin{aligned}
& \left\langle G_{0}\left(\tau_{1}\right) G_{0}\left(\tau_{2}\right)\right\rangle\left\langle G_{0}\left(\tau_{3}\right) G_{2}\left(\tau_{4}\right)\right\rangle, \\
& \left\langle G_{0}\left(\tau_{1}\right) G_{0}\left(\tau_{2}\right)\right\rangle\left\langle G_{1}\left(\tau_{3}\right) G_{1}\left(\tau_{4}\right)\right\rangle, \\
& \left\langle G_{0}\left(\tau_{1}\right) G_{0}\left(\tau_{2}\right) G_{1}\left(\tau_{3}\right)\right\rangle\left\langle G_{1}\left(\tau_{4}\right)\right\rangle, \\
& \left\langle G_{0}\left(\tau_{1}\right) G_{0}\left(\tau_{2}\right)\right\rangle\left\langle G_{1}\left(\tau_{3}\right)\right\rangle\left\langle G_{1}\left(\tau_{4}\right)\right\rangle .
\end{aligned}
$$

Clearly, the terms of maximal order in cumulants corresponds to the case when all indices $i_{s}$ in Eqs. (24) and (25) are equal to one. Then the cumulant expansion of all such terms contains the contribution 


$$
\left\langle G_{0} G_{0}\right\rangle\left\langle G_{1}\right\rangle\left\langle G_{1}\right\rangle \cdots\left\langle G_{1}\right\rangle,
$$

which contains $l$ factors $\left\langle G_{1}\left(t_{i}\right)\right\rangle$ and, therefore, is of order $l+1$ in cumulants. However, all terms of order $l+2$ in $G_{i}$ described above enter in $C_{2 l}$ via combinations

$$
\begin{aligned}
& \left\langle G_{0} G_{0} G_{i_{1}} G_{i_{2}} \cdots G_{i_{l}}\right\rangle-\left\langle G_{0} G_{0}\right\rangle\left\langle G_{i_{1}} G_{i_{2}} \cdots G_{i_{l}}\right\rangle \\
& \left\langle G_{0} G_{0} G_{i_{1}}\right\rangle\left\langle G_{i_{2}} \cdots G_{i_{l}}\right\rangle-\left\langle G_{0} G_{0}\right\rangle\left\langle G_{i_{1}}\right\rangle\left\langle G_{i_{2}} \cdots G_{i_{l}}\right\rangle \\
& \left\langle G_{0} G_{0} G_{i_{1}} G_{i_{2}}\right\rangle\left\langle G_{i_{3}} \cdots G_{i_{l}}\right\rangle-\left\langle G_{0} G_{0}\right\rangle\left\langle G_{i_{1}} G_{i_{2}}\right\rangle\left\langle G_{i_{3}} \cdots G_{i_{l}}\right\rangle, \cdots
\end{aligned}
$$

which is a consequence of a presence of the operator $\mathcal{Q}$ (first from the left) in the definition of $C_{i}$ (see Eq. (14)). As a result, the contributions (27) involving $l+1$ cumulant factors cancel, and the maximal order in cumulants of surviving terms in the expression for $C_{2 l}$ contain at most $l$ cumulant factors.

Having established that the contributions to $C_{2 l}$ from terms of maximal order $l+2$ in $G_{i}$ are of order $l$ in cumulants, it is clear that the terms of order $l+1$ and lower in $G_{i}$ cannot contain more that $l$ cumulant factors since such terms contain at least one isolated factor of $G_{0}$ whose average vanishes. Hence $C_{2 l}$ contains at most $l$ cumulant factors.

In the next section, we examine the consequences of the cumulant expansions of the memory function for a specific system, namely, the Rayleigh model with a repulsive parabolic potential. It will be demonstrated that for the Rayleigh model, cumulants are linear functions of the average number $N$ of particles in the interaction zone around the particle. This, in turn, implies that the terms in the $\lambda$-expansion behave as

$$
K_{2 l} \sim\left(N+N^{2}+\cdots+N^{l}\right) \lambda^{2 l}
$$

for $l>1$. Clearly, for a large particle and/or long-ranged potential leading to $N \gg 1$, these results suggest that $K_{2 l} \sim N^{l} \lambda^{2 l}$, demonstrating that the actual parameter of $\lambda$-expansion for this exactly solvable model is in fact $N^{1 / 2} \lambda$. On the other hand, for a short-ranged potential, when $N \ll 1$, all terms in the expansion are linear in $N$, and one sees that $K_{2 l} \sim N \lambda^{2 l}$, and the effective small parameter of $\lambda$-expansion is in fact the square-root of the mass ratio. To further illustrate this analysis, the explicit form of the cumulant expansion of $C_{4}$ is presented in Appendix A.

\section{THE LINEAR LANGEVIN EQUATION FOR A HEAVY PARTICLE OR IDEALIZED PISTON}

Consider the random motion of a piston of mass $M$ and cross-sectional area $S$ subjected to collisions with an ideal gas particles of mass $m$. The gas particles and the piston are constrained to move in one dimension perpendicular to the piston faces. The velocity distribution of incident particles $f_{M}(v)$ before collision with the piston is Maxwellian with inverse temperature $\beta$, namely

$$
f_{M}(v)=\left(\frac{m \beta}{2 \pi}\right)^{1 / 2} \exp \left(-\frac{1}{2} \beta m v^{2}\right) .
$$

The piston-particle interaction is assumed to be described by a purely repulsive parabolic potential. For particles to the left of the piston the interaction potential between a gas particle and the piston is

$$
U_{l}= \begin{cases}\frac{1}{2} k_{f}\left(x-X_{l}\right)^{2} & x>X_{l}, \\ 0 & x<X_{l},\end{cases}
$$

where $k_{f}$ is a force constant, $x$ is the coordinate of the gas particle, $X_{l}=X_{l f}-a$ the boundary of the piston-particle interaction zone, $X_{l f}$ is the coordinate of the left face of the piston, and $a$ is the width of the interaction zone. Similarly, the gas particle-piston potential for the particles to the right of the piston has the analogous form

$$
U_{r}= \begin{cases}\frac{1}{2} k_{f}\left(x-X_{r}\right)^{2} & x<X_{r}, \\ 0 & x>X_{r},\end{cases}
$$

where $X_{r}=X_{r f}+a$, and $X_{r f}$ is the position of the piston's right face. We assume that the temperature is low enough (or $k_{f}$ is sufficiently large) so that the probability for a particle to reach the piston's surface is negligible.

In the previous sections it was established that the dynamics of the piston can be deduced from time correlation functions describing molecular motion in the field of the piston fixed in space. Below we derive the explicit expressions for these correlation functions in the thermodynamic limit, neglecting recollisions of the piston and gas particles due to the finite size of the bath. 
Consider the force on the left side of the fixed piston,

$$
F_{l}(t)=-k_{f} \sum_{i} q_{i}(t) \theta\left(q_{i}(t)\right)
$$

where $q_{i}=x_{i}-X_{l}$ is the position of gas particle $i$ relative to the boundary of the interaction zone, $\theta(x)$ is the step-function, and summation over index $i$ is over all particle in the tube of diameter $S$ to the left of the piston. In this section we omit for brevity the subscript 0 for the force on the fixed piston. The simplifying feature of parabolic potential is that the time $\tau_{c}$ that a particle spends in the interaction zone of the fixed piston is independent of the initial velocity of the particle and given by $\tau_{c}=\pi / \omega$, where $\omega=\sqrt{k_{f} / m}$. At a given time $t$, the only gas particles in the interaction zone are those that had positive velocities and coordinates $q$ in the interval $-v \tau_{c}<q<0$ at time $t-\tau_{c}$. At time $t$, the position of the gas particle is determined by $q(t)=(v / \omega) \sin \omega\left(t-t_{\text {in }}\right)$, where $t_{\text {in }}=t-\tau_{c}-q / v$ corresponds to the time at which the gas particle enters the interaction region and $q$ is the position of the gas particle at time $t-\tau_{c}$. It then follows that $q(t)=(v / \omega) \sin \omega\left(\tau_{c}+q / v\right)=-(v / \omega) \sin \omega q / v$, which implies that the total instantaneous force on the left side of the fixed piston at time $t>0$ can be written as

$$
F_{l}(t)=-k_{f} \int_{0}^{\infty} d v \int_{-v \tau_{c}}^{0} d q N\left(X_{l}+q, v ; t-\tau_{c}\right) \frac{v}{\omega} \sin \frac{\omega q}{v} .
$$

In Eq. (33), $q=x-X_{l}$ and $N(x, v ; t)$ is the microscopic linear density of particles defined by

$$
N(x, v ; t)=\sum_{i} \delta\left(x-x_{i}(t)\right) \delta\left(v-v_{i}(t)\right) .
$$

Similarly, the total instantaneous force acting on the right side of the piston is

$$
F_{r}(t)=-k_{f} \int_{-\infty}^{0} d v \int_{0}^{-v \tau_{c}} d q N\left(X_{r}+q, v ; t-\tau_{c}\right) \frac{v}{\omega} \sin \frac{\omega q}{v}
$$

where $q=x-X_{r}$.

For a particle outside the interaction zone of the fixed piston (i.e. for $x<X_{l}$, and $x>X_{r}$ ), the average linear density of particles is $\langle N(x, v)\rangle=n S f_{M}(v)$, where $n$ is the total (three-dimensional) density of bath particles, and $S$ is the cross-sectional area of the piston. It then follows from Eqs. (33) and (35) that the average force acting on the left $\left\langle F_{l}\right\rangle$ and the right $\left\langle F_{r}\right\rangle$ sides of the piston are $\left\langle F_{l}\right\rangle=-\left\langle F_{r}\right\rangle=n S / \beta$.

It is straightforward to show that the stationary distribution in the vicinity of the fixed piston, including the interaction zone, assumes Boltzmann's form

$$
\langle N(x, v)\rangle=n S f_{M}(v) \exp \left(-U(x) / k_{B} T\right) .
$$

To calculate the force correlation functions required to analyze the damping terms in the LE, correlation functions of the form $\left\langle N\left(Y_{1}\right) N\left(Y_{2}\right) \cdots N\left(Y_{s}\right)\right\rangle$ must be evaluated, where $Y$ denotes the position-velocity pair $(x, v)$. It is sufficient to consider only the case when time arguments are equal for all functions, since time displacement can be transformed into spatial displacement for a free particle, i.e.

$$
N\left(x, v ; t+t_{1}\right)=N\left(x-v t_{1}, v ; t\right) .
$$

Note that the product $N\left(Y_{1}\right) N\left(Y_{2}\right)$ can be written as

$$
\begin{aligned}
N\left(Y_{1}\right) N\left(Y_{2}\right) & =\sum_{i, j} \delta\left(Y_{1}-Y_{i}\right) \delta\left(Y_{2}-Y_{j}\right) \\
& =\sum_{i} \delta\left(Y_{1}-Y_{i}\right) \delta\left(Y_{2}-Y_{i}\right)+\sum_{i \neq j} \delta\left(Y_{1}-Y_{i}\right) \delta\left(Y_{2}-Y_{j}\right) .
\end{aligned}
$$

Since $\delta\left(Y_{1}-Y_{i}\right) \delta\left(Y_{2}-Y_{i}\right)=\delta\left(Y_{1}-Y_{2}\right) \delta\left(Y_{1}-Y_{i}\right)$, for the ideal gas system one obtains

$$
\left\langle N\left(Y_{1}\right) N\left(Y_{2}\right)\right\rangle=\delta\left(Y_{1}-Y_{2}\right)\left\langle N\left(Y_{1}\right)\right\rangle+\left\langle N\left(Y_{1}\right)\right\rangle\left\langle N\left(Y_{2}\right)\right\rangle .
$$

For the three-point correlation function, the same arguments lead to the result 


$$
\begin{aligned}
\left\langle N\left(Y_{1}\right) N\left(Y_{2}\right) N\left(Y_{3}\right)\right\rangle & =\delta\left(Y_{1}-Y_{2}\right) \delta\left(Y_{2}-Y_{3}\right)\left\langle N\left(Y_{1}\right)\right\rangle \\
& +\delta\left(Y_{1}-Y_{2}\right)\left\langle N\left(Y_{1}\right)\right\rangle\left\langle N\left(Y_{3}\right)\right\rangle+\delta\left(Y_{1}-Y_{3}\right)\left\langle N\left(Y_{1}\right)\right\rangle\left\langle N\left(Y_{2}\right)\right\rangle \\
& +\delta\left(Y_{2}-Y_{3}\right)\left\langle N\left(Y_{1}\right)\right\rangle\left\langle N\left(Y_{2}\right)\right\rangle+\left\langle N\left(Y_{1}\right)\right\rangle\left\langle N\left(Y_{2}\right)\right\rangle\left\langle N\left(Y_{3}\right)\right\rangle .
\end{aligned}
$$

Eqs. (38) and (39) are the cumulant expansions of $\left\langle N\left(Y_{1}\right) N\left(Y_{2}\right)\right\rangle$ and $\left\langle N\left(Y_{1}\right) N\left(Y_{2}\right) N\left(Y_{3}\right)\right\rangle$, where the cumulants

$$
\begin{aligned}
\left\langle\left\langle N\left(Y_{1}\right) N\left(Y_{2}\right)\right\rangle\right\rangle & =\delta\left(Y_{1}-Y_{2}\right)\left\langle N\left(Y_{1}\right)\right\rangle \\
\left\langle\left\langle N\left(Y_{1}\right) N\left(Y_{2}\right) N\left(Y_{3}\right)\right\rangle\right\rangle & =\delta\left(Y_{1}-Y_{2}\right) \delta\left(Y_{2}-Y_{3}\right)\left\langle N\left(Y_{1}\right)\right\rangle \\
\left\langle\left\langle N\left(Y_{1}\right) N\left(Y_{2}\right) \cdots N\left(Y_{s}\right)\right\rangle\right\rangle & =\delta\left(Y_{1}-Y_{2}\right) \delta\left(Y_{2}-Y_{3}\right) \cdots \delta\left(Y_{s-1}-Y_{s}\right)\left\langle N\left(Y_{1}\right)\right\rangle
\end{aligned}
$$

are proportional to the equilibrium density $n$ of gas particles.

To order $\lambda^{2}$, the dynamics of the piston is described by the LE (17) with a time-dependent damping coefficient, $\gamma_{0}(t)=\frac{\beta}{m} \int_{0}^{t} d \tau\left\langle F F_{0}(\tau)\right\rangle$, where the evolution of the total force $F_{0}=F_{l}+F_{r}$ is determined by the constrained piston-bath Liouville operator $\mathcal{L}_{0}$. Since $\left\langle F_{l}\right\rangle=-\left\langle F_{r}\right\rangle$ and $\left\langle F_{l} F_{l}(t)\right\rangle=\left\langle F_{r} F_{r}(t)\right\rangle$,

$$
\left\langle F F_{0}(t)\right\rangle=2\left\langle F_{l} F_{l}(t)\right\rangle-2\left\langle F_{l}\right\rangle^{2}=2\left\langle\left\langle F_{l} F_{l}(t)\right\rangle\right\rangle .
$$

From Eqs. (41) and (33), one sees that $\left\langle F F_{0}(t)\right\rangle$ can be expressed in terms of the cumulant $\langle\langle N(x, v ; t-$ $\left.\left.\left.\tau_{c}\right) N\left(x^{\prime}, v^{\prime} ;-\tau_{c}\right)\right\rangle\right\rangle$. Using property (37) and Eq. (41), the cumulant may be rewritten as

$$
\left\langle\left\langle N\left(x-v t, v ;-\tau_{c}\right) N\left(x^{\prime}, v^{\prime} ;-\tau_{c}\right)\right\rangle=\delta\left(x-v t-x^{\prime}\right) \delta\left(v-v^{\prime}\right) n S f_{M}(v) .\right.
$$

Then, using Eqs. (41), (33) and (42), one obtains

$$
\left\langle F F_{0}(t)\right\rangle=\frac{n S k_{f}^{2}}{\omega^{3}} \theta\left(\tau_{c}-t\right)\left\{\sin \omega t+\omega\left(\tau_{c}-t\right) \cos \omega t\right\} \int_{0}^{\infty} d v f_{M}(v) v^{3} .
$$

This can be re-expressed in the compact form

$$
\left\langle F F_{0}(t)\right\rangle=N \omega^{2} \frac{m}{\beta} \xi_{0}(t),
$$

where $\xi_{0}(t)$ is a dimensionless function (see Fig. 1) given by

$$
\xi_{0}(t)=\sqrt{\frac{2}{\pi}} \theta\left(\tau_{c}-t\right)\{\sin \omega t+(\pi-\omega t) \cos \omega t\}
$$

and

$$
N=n S \frac{\left\langle v^{2}\right\rangle^{\frac{1}{2}}}{\omega}
$$

is the average number of particles in the shell of thickness $l=\sqrt{\left\langle v^{2}\right\rangle} / \omega$ around the piston. The parameter $l$ specifies the length at which the average bath particle penetrates the interaction zone, and $N=n S l$ is the average number of particles in the layer of thickness $l$ around the piston. Thus, it is evident that in addition to the mass ratio $\lambda^{2}=m / M$, the relevant physics depends strongly on another characteristic parameter of the system, namely, $N \lambda^{2}$, which can be interpreted as the ratio of total mass $M_{*}=m n S l$ of bath particles in the layer of thickness $l$ in the vicinity of the piston to the mass $M$ of the piston.

With these definitions in hand, the time dependent damping coefficient in the LE (17) can be written as

$$
\gamma_{0}(t)=\frac{\beta}{m} \int_{0}^{t} d \tau\left\langle F F_{0}(t)\right\rangle=\omega N \zeta_{0}(t),
$$

and therefore the LE (17) assumes the form

$$
\frac{d P_{*}(t)}{d t}=\lambda F^{\dagger}(t)-\omega \lambda^{2} N \zeta_{0}(t) P_{*}(t),
$$

where the damping function $\zeta_{0}(t)=\omega \int_{0}^{t} d \tau \xi_{0}(\tau)$ is given by 


$$
\zeta_{0}(t)=\sqrt{\frac{2}{\pi}} \theta\left(\tau_{c}-t\right)\{2(1-\cos \omega t)+(\pi-\omega t) \sin \omega t\}+4 \sqrt{\frac{2}{\pi}} \theta\left(t-\tau_{c}\right)
$$

(see Fig.1). This expression describes the interesting time-development of the dissipative force, an aspect of the dynamics that is outside the scope of more phenomenological models. For $t<\tau_{c}$, Eq. (48) describes essentially non-exponential relaxation of the momentum $\langle P(t)\rangle=P(0) e^{-\chi(t)}$ with

$$
\chi(t)=\lambda^{2} N \omega \int_{0}^{t} d \tau \zeta_{0}(\tau)=\lambda^{2} N \sqrt{\frac{2}{\pi}}\{\pi+2 \omega t-(\pi-\omega t) \cos \omega t-3 \sin \omega t\} .
$$

For small $\omega t, \chi(t) \sim(\omega t)^{2}$. On a time-scale $t>\tau_{c}$ the damping function reaches its plateau value and the Markovian limit of the Langevin equation, in which the damping coefficient is independent of time, is recovered:

$$
\frac{d P_{*}(t)}{d t}=\lambda F^{\dagger}(t)-4 \sqrt{\frac{2}{\pi}} \omega \lambda^{2} N P_{*}(t)
$$

Note that the characteristic time for relaxation of the momentum $\tau_{p}=\omega^{-1}\left(\lambda^{2} N\right)^{-1}$ is governed by the parameter $\lambda^{2} N$, rather than $\lambda^{2}$. It is also interesting to observe that the average number of collisions of bath particles with the piston for $t \sim \tau_{p}$ is of order $n S\left\langle v^{2}\right\rangle^{1 / 2} \tau_{p}=\lambda^{-2}$ and depends neither on temperature, nor on the piston's size, but only on the mass ratio.

Equation (51) is obtained under the condition that the characteristic time $\tau_{c}$ for the force autocorrelation function is negligible on a time scale $\tau_{p}$ of dynamics of the momentum of the piston. Assuming in addition that the random force in this equation is Gaussian, one can obtain the Fokker-Planck equation for the momentum distribution function $f(P)$

$$
\frac{\partial f(P)}{\partial t}=n S \frac{4 m}{M} \sqrt{\frac{2 k_{B} T}{\pi m}}\left\{\frac{\partial}{\partial P}(P f(P))+M k_{B} T \frac{\partial^{2} f(P)}{\partial P^{2}}\right\} .
$$

This coincides exactly with the equation for the piston interacting with the bath particles through a hard-wall potential previously obtained by Van Kampen from the master equation [10].

The assumption of a Gaussian random force appears to be justified for Eq. (51) describing dynamics on time scales much longer than $\tau_{c}$. In this case one can use a coarse-grained description of the dynamics with time resolution $\tau_{c} \ll \Delta t \ll \tau_{p}$. The coarse-graining procedure corresponds to replacing the instantaneous random force in Eq. (51) by its average over a time window of duration $\Delta t$, i.e. $F(t) \rightarrow \hat{F}(t)=\Delta t^{-1} \int_{t}^{t+\Delta t} F(t) d t$. As previously discussed, the number of collisions of bath particles with the piston for the time interval $\tau_{p}$ is of order $\lambda^{-2} \gg 1$. Therefore the resolution time interval $\Delta t$ may be chosen sufficiently long that the piston experiences many uncorrelated collisions during $\Delta t$. Then, according the central limit theorem, one may expect that $\hat{F}(t)$ is Gaussian-distributed.

For the more general LE (17) with time-dependent damping, the random force is generally not Gaussian-distributed. However, one can easily demonstrate that the distribution of the random force is approximately Gaussian in the limit $N \gg 1$ where the piston interact simultaneously with many bath particles. In fact, the cumulant expansion (23) of the multi-time correlation function $C_{2 s}=\left\langle F\left(t_{1}\right) F\left(t_{2}\right) \cdots F\left(t_{2 s}\right)\right\rangle$ contains the products of $s$ pair correlation functions $\left\langle F\left(t_{i}\right) F\left(t_{j}\right)\right\rangle=\left\langle\left\langle F\left(t_{i}\right) F\left(t_{j}\right)\right\rangle\right\rangle$. Since a cumulant of any order is proportional $N$, these terms are of order $N^{s}$. The other terms in the expansion contain fewer factors of the cumulants and therefore fewer factors of $N$, and hence may be neglected. Then $C_{2 s}$ can be approximately expressed as a linear combination of pair correlation functions, a wellknown characteristic of a Gaussian random variable. The explicit form of the distribution function for the random force $f\left(F_{0}\right)$ can be obtained using the inverse Fourier transformation of the generating function (see, for example, reference $[2])$

$$
f\left(F_{0}\right)=\frac{1}{2 \pi} \int_{-\infty}^{\infty} d k \exp \left\{-i k F_{0}+\sum_{s=1}^{\infty} \frac{(i k)^{s}}{s !}\left\langle\left\langle F_{0}^{s}\right\rangle\right\rangle .\right.
$$

The cumulants of odd orders vanish, and the cumulant of even orders are $\left\langle\left\langle F_{0}^{2 s}\right\rangle\right\rangle=2\left\langle\left\langle F_{l}^{2 s}\right\rangle\right\rangle$ where $F_{l}$ is given by (33). Using (41) one finds that

$$
\left\langle\left\langle F_{l}^{2 s}\right\rangle\right\rangle=\frac{1}{\sqrt{2}} N g^{2 s} s ! \frac{\Gamma(s+1 / 2)}{\Gamma(s+1)},
$$

where $g^{2}=k_{f}^{2}\left\langle v^{2}\right\rangle / 2 \omega^{2}$, and $\Gamma(s)$ is the gamma-function. Substitution (54) in (53) gives the following integral representation for the distribution function 


$$
f(F)=\frac{1}{2 \pi} \int_{-\infty}^{\infty} d k \exp \left\{-i k F-\sqrt{2 \pi} N\left(1-e^{-(g k)^{2}}\right)\right\}
$$

If $N \gg 1$, one can approximately write in the above expression $1-e^{-(g k)^{2}} \approx(g k)^{2}$, which leads immediately to the Gaussian distribution for the force. The similar arguments hold for the distribution function of higher order $f\left(F\left(t_{1}\right), F\left(t_{2}\right), \cdots F\left(t_{s}\right)\right)$.

\section{THE NONLINEAR LANGEVIN EQUATION}

We now turn our attention to the terms of higher order in $\lambda$ in the $\lambda$-expansion (13) of the memory function $K(t) \equiv\left\langle F F^{\dagger}(t)\right\rangle=\sum_{l} K_{l}(t)$. It was shown in section 3 that for a homogeneous bath $K_{1}(t)=0$, and the first non-zero correction to $K_{0}(t)=\left\langle F F_{0}(t)\right\rangle$ is $K_{2}(t)$ which is of second order in $\lambda$. From Eqs. (13) and (23) one can see that $K_{2}$ has the structure

$$
K_{2}(t)=\frac{\lambda^{2}}{m^{2}} A_{1}(t) P_{*}^{2}+\frac{\lambda^{2}}{m} A_{2}(t)
$$

where the functions $A_{1}(t)$ and $A_{2}(t)$ are given by

$$
\begin{aligned}
& A_{1}(t)=\int_{0}^{t} d t_{1} \int_{0}^{t_{1}} d t_{2}\left\langle\left\langle G_{0} G_{2}\left(t, t_{1}, t_{2}\right)\right\rangle\right\rangle, \\
& A_{2}(t)=\int_{0}^{t} d t_{1} \int_{0}^{t_{1}} d t_{2}\left\langle\left\langle G_{0} G_{0}\left(t-t_{1}\right) G_{1}\left(t, t_{2}\right)\right\rangle\right\rangle .
\end{aligned}
$$

Substitution of $K \approx K_{0}+K_{2}$ into the exact equation of motion (11) leads to the non-linear generalized LE of the form

$$
\frac{d P_{*}(t)}{d t}=\lambda F^{\dagger}(t)-\lambda^{2} \int_{0}^{t} d \tau M_{1}(\tau) P_{*}(t-\tau)-\lambda^{4} \int_{0}^{t} d \tau M_{2}(\tau) P_{*}^{3}(t-\tau)
$$

where the memory functions $M_{1}(t)$ and $M_{2}(t)$ are

$$
\begin{aligned}
& M_{1}(t)=M_{0}(t)-\frac{2 \lambda^{2}}{m^{2}} A_{1}(t)+\frac{\lambda^{2} \beta}{m^{2}} A_{2}(t), \\
& M_{2}(t)=\frac{\beta}{m^{3}} A_{1}(t) .
\end{aligned}
$$

It is interesting to note that a nonlinear LE of this form has previously been obtained using a mode coupling approach [15]. A similar Markovian version of a nonlinear Langevin equation with a cubic damping term was considered by MacDonald [16] on a purely phenomenological grounds.

Eq. (59) differs from the linear LE (15) with memory function $M_{0}(t)=\frac{\beta}{m}\left\langle F F_{0}(t)\right\rangle$ not only by the presence of nonlinear damping, but also by appearance of correction terms of order $\lambda^{2}$ to the memory function $M_{1}(t)$ for the linear damping. Note also that the last term on the right-hand side of (59) can be written in the local form $-\lambda^{4} P_{*}^{3}(t) \int_{0}^{t} d \tau M_{2}(\tau)$ since the non-local correction to this expression $\lambda^{4} \int_{0}^{t} d \tau M_{2}(\tau) \int_{t-\tau}^{t} d \tau^{\prime} \dot{P_{*}^{3}}\left(\tau^{\prime}\right)$ is of order $\lambda^{5}$ [14]. However, the linear damping term in Eq. (59) cannot be simply written in the local form $-\lambda^{2} P_{*}(t) \int_{0}^{t} d \tau M_{1}(\tau)$ since in this case the non-local correction has contributions of order $\lambda^{3}$ and $\lambda^{4}$ which must be retained. This correction can be written in the form

$$
\begin{aligned}
& \lambda^{2} \int_{0}^{t} d \tau M_{1}(\tau) \int_{t-\tau}^{t} d \tau^{\prime} \dot{P}_{*}\left(\tau^{\prime}\right)=\lambda^{3} \int_{0}^{t} d \tau M_{0}(\tau) \int_{t-\tau}^{t} d \tau^{\prime} F^{\dagger}\left(\tau^{\prime}\right) \\
& -\lambda^{4} \int_{0}^{t} d \tau M_{0}(\tau) \int_{t-\tau}^{t} d \tau^{\prime} \int_{0}^{\tau^{\prime}} d \tau^{\prime \prime} M_{0}\left(\tau^{\prime \prime}\right) P_{*}\left(\tau^{\prime}-\tau^{\prime \prime}\right)+O\left(\lambda^{5}\right)
\end{aligned}
$$

where we have used the result that $M_{1}(t)=M_{0}(t)+O\left(\lambda^{2}\right)$ according to $(60)$. The first term in the right-hand side of this expression depends on initial coordinates of the bath and may be treated as a small correction to the random force $F^{\dagger}(t)$. The second term can be written in the local form 


$$
-\lambda^{4} P_{*}(t) \int_{0}^{t} d \tau M_{0}(\tau) \int_{t-\tau}^{t} d \tau^{\prime} \int_{0}^{\tau^{\prime}} d \tau^{\prime \prime} M_{0}\left(\tau^{\prime \prime}\right)+O\left(\lambda^{5}\right)
$$

to order $\lambda^{5}$. As a result, Eq. (59) can be written in the local form

$$
\frac{d P_{*}(t)}{d t}=\lambda \tilde{F}^{\dagger}(t)-\lambda^{2} \gamma_{1}(t) P_{*}(t)-\lambda^{4} \gamma_{2}(t) P_{*}^{3}(t),
$$

with the modified random force

$$
\tilde{F}^{\dagger}(t)=F^{\dagger}(t)+\lambda^{3} \int_{0}^{t} d \tau M_{0}(\tau) \int_{t-\tau}^{t} d \tau^{\prime} F^{\dagger}\left(\tau^{\prime}\right)
$$

and the damping functions given by

$$
\begin{aligned}
& \gamma_{1}(t)=\int_{0}^{t} d \tau M_{1}(\tau)+\lambda^{2} \int_{0}^{t} d \tau M_{0}(\tau) \int_{t-\tau}^{t} d \tau^{\prime} \int_{0}^{\tau^{\prime}} d \tau^{\prime \prime} M_{0}\left(\tau^{\prime \prime}\right), \\
& \gamma_{2}(t)=\int_{0}^{t} d \tau M_{2}(\tau) .
\end{aligned}
$$

For $t>>\tau_{c}$, the time-dependent coefficients $\gamma_{i}(t)$ attain their limiting time-independent values $\gamma_{i}$, which can be obtained from the expressions above by setting the upper integration limit $t$ to infinity.

It is possible to obtained explicit expressions for the memory functions $M_{i}(t)$ and the damping functions $\gamma_{i}(t)$ for the extended Rayleigh model. To accomplish this, explicit expressions for the functions $G_{i}$ defined by Eqs.(19) must be computed. It is convenient to express these functions as the sum of two parts corresponding to the force acting on left and right sides of the piston, $G_{i}=G_{l i}+G_{r i}$. It is somewhat problematic to calculate terms such as $G_{l 1}=e^{\mathcal{L}_{0} \tau} \partial F_{l}(t) / \partial X$ due to the parametric dependence of $F_{l}(t)$ on $X$ that is evident when the force is expressed in terms of $q_{i}(t)$ and $v_{i}(t)$ (see Eq. (33)). One straightforward, albeit inelegant, way to circumvent this difficulty is to express the force in terms of $N(x, v) \equiv N(x, v ; t=0)$. Details of this technique can be found in the Appendix B. Using this approach, we obtain for the left-side part of $G_{1}$

$$
\begin{aligned}
G_{l 1}\left(t_{1}, t_{2}\right)=k_{f} \int_{0}^{\infty} d v & \int_{-v\left(\tau_{c}+t_{1}\right)}^{b} d q N\left(X_{l}+q, v ;-\tau_{c}\right) \cos \omega\left(t_{1}+\frac{q}{v}\right) \\
& -k_{f} \theta\left(\tau_{c}-t_{2}\right) \cos \omega t_{2} \int_{0}^{\infty} d v \int_{-v\left(\tau_{c}+t_{1}-t_{2}\right)}^{-v t_{1}} d q N\left(X_{l}+q, v ;-\tau_{c}\right),
\end{aligned}
$$

where the integration limit $b$ is

$$
b=-v\left(\tau_{c}+t_{1}-t_{2}\right) \theta\left(\tau_{c}-t_{2}\right)-v t_{1} \theta\left(t_{2}-\tau_{c}\right) .
$$

The expression for $G_{r 1}$ can be obtained from the one above by replacements,

$$
\int_{0}^{\infty} d v \rightarrow \int_{-\infty}^{0} d v, \quad \int_{q_{1}}^{q_{2}} d q \rightarrow \int_{q_{2}}^{q_{1}} d q
$$

Note that $\left\langle G_{l 1}\right\rangle=\left\langle G_{r 1}\right\rangle$.

For the left-side contribution to $G_{2}$, we obtain

$$
\begin{aligned}
G_{l 2}\left(t, t_{1}, t_{2}\right)=k_{f} \int_{0}^{\infty} d v N\left(X_{l}-v\left(t+\tau_{c}\right), v ;-\tau_{c}\right) & \\
+k_{f} \int_{0}^{\infty} d v \int_{-v\left(t+\tau_{c}\right)}^{b} d q N\left(X_{l}+q, v ;-\tau_{c}\right) \frac{\omega}{v} \sin \omega\left(t+\frac{q}{v}\right) & +k_{f} \phi\left(t_{1}, t_{2}\right) \int_{0}^{\infty} d v N\left(X_{l}-v t, v ;-\tau_{c}\right),
\end{aligned}
$$


where the function $\phi\left(t, t_{1}, t_{2}\right)$ is

$$
\begin{aligned}
\phi\left(t_{1}, t_{2}\right) & =\theta\left(t_{2}-\tau_{c}\right)-\theta\left(t_{1}-\tau_{c}\right) \theta\left(\tau_{c}-t_{2}\right) \cos \omega t_{2} \\
& +\theta\left(\tau_{c}-t_{1}\right) \theta\left(\tau_{c}-t_{2}\right) \cos \omega t_{2} \cos \omega t_{1},
\end{aligned}
$$

and the upper integration limit $b$ is

$$
b=-v\left(\tau_{c}+t-t_{2}\right) \theta\left(\tau_{c}-t_{2}\right)-v t \theta\left(t_{2}-\tau_{c}\right) .
$$

A similar expression for $G_{r 2}$ can be obtained from that for $G_{l 2}$ using replacements (70), and, in addition, by multiplying the first and the last terms on the right-hand side of Eq. (71) by -1 . Recall that we anticipate $\left\langle G_{2}\right\rangle=0$ by symmetry so that $\left\langle G_{r 2}\right\rangle=-\left\langle G_{l 2}\right\rangle$, which can be explicitly verified from the expressions above.

It has been shown above that the damping forces in the nonlinear Langevin equation can be expressed as integrals of the cumulants $\left\langle\left\langle G_{0} G_{2}\right\rangle\right\rangle$ and $\left\langle\left\langle G_{0} G_{0} G_{1}\right\rangle\right\rangle$. From Eqs. (68), (71), (33), and (41), one can get an explicit expressions for these cumulants. For $t>t_{1}>t_{2}$ we find

$$
\begin{aligned}
\left\langle\left\langle G_{0} G_{2}\left(t, t_{1}, t_{2}\right)\right\rangle\right\rangle & =2\left\langle\left\langle G_{l 0} G_{l 2}\left(t, t_{1}, t_{2}\right)\right\rangle\right\rangle \\
& =\frac{n S k_{f}^{2}}{\omega} \phi_{1}\left(t, t_{1}, t_{2}\right) \int_{0}^{\infty} d v f_{M}(v) v,
\end{aligned}
$$

where

$$
\phi_{1}\left(t, t_{1}, t_{2}\right)=2 \theta\left(\tau_{c}-t\right) \sin \omega t \cos \omega t_{1} \cos \omega t_{2} .
$$

The second cumulant required is

$$
\begin{aligned}
\left\langle\left\langle G_{0} G_{0}\left(t-t_{1}\right) G_{1}\left(t, t_{2}\right)\right\rangle\right\rangle & =2\left\langle\left\langle G_{l 0} G_{l 0}\left(t-t_{1}\right) G_{l 1}\left(t, t_{2}\right)\right\rangle\right\rangle \\
& =-n S\left(\frac{k_{f}}{\omega}\right)^{3} \phi_{2}\left(t, t_{1}, t_{2}\right) \int_{0}^{\infty} d v f_{M}(v) v^{3},
\end{aligned}
$$

where the function $\phi_{2}$ has the form

$$
\phi_{2}\left(t, t_{1}, t_{2}\right)=\theta\left(\tau_{c}-t\right) \cos \omega t_{2} \times
$$

$$
\left\{\omega\left(\tau_{c}-t\right) \cos \omega\left(t-t_{1}\right)+\frac{1}{2} \sin \omega\left(t+t_{1}\right)+\frac{1}{2} \sin \omega\left(t-t_{1}\right)\right\}
$$

These results allow to calculate the functions $A_{i}(t)$ defined by Eqs. (57) and (58),

$$
\begin{aligned}
& A_{1}(t)=m^{2} \omega^{2} N \xi_{1}(t), \\
& A_{2}(t)=-m^{2} \omega^{2} \beta^{-1} N \xi_{2}(t),
\end{aligned}
$$

where dimensionless functions $\xi_{i}(t)$ are given by

$$
\begin{aligned}
& \xi_{1}(t)=\frac{1}{\sqrt{2 \pi}} \theta\left(\tau_{c}-t\right) \sin ^{3} \omega t, \\
& \xi_{2}(t)=\frac{1}{\sqrt{2 \pi}} \theta\left(\tau_{c}-t\right)\left\{\sin ^{3} \omega t+\omega t(\pi-\omega t) \sin \omega t\right\} .
\end{aligned}
$$

The memory functions in the non-Markovian LE equation (59), take the form

$$
\begin{aligned}
& M_{1}(t)=N \omega^{2}\left\{\xi_{0}(t)-2 \lambda^{2} \xi_{1}(t)-\lambda^{2} \xi_{2}(t)\right\}, \\
& M_{2}(t)=N \omega^{2} \frac{\beta}{m} \xi_{1}(t),
\end{aligned}
$$

Here the function $\xi_{0}(t)$ is given by $(45)$ and governs the memory function $M_{0}(t)$ in the linear LE $(15), M_{0}(t)=$ $N \omega^{2} \xi_{0}(t)$. The additional $\lambda^{2}$-correction terms leads to a faster decay of the linear damping kernel $M_{1}(t)$ compared to $M_{0}(t)$. The kernel for nonlinear damping $M_{2}(t)$ is not decaying function of time but rather has a maximum at $t=\tau_{c} / 2$. 
Explicit expressions for damping functions $\gamma_{i}(t)$ in the local-in-time LE (64) by integrating $M_{i}(t)$ according to Eqs. (66) and (67), and the nonlinear LE for the Rayleigh model with a parabolic potential takes the form

$$
\frac{d P_{*}(t)}{d t}=\lambda \tilde{F}^{\dagger}(t)-N \lambda^{2} \omega\left\{\zeta_{1}(t) P_{*}(t)+\lambda^{2} \frac{\beta}{m} \zeta_{2}(t) P_{*}^{3}(t)\right\},
$$

where the nonlinear damping function $\zeta_{2}(t)$ is given by

$$
\zeta_{2}(t)=\omega \int_{0}^{t} d \tau \xi_{1}(\tau)=\frac{1}{\sqrt{2 \pi}} \theta\left(\tau_{c}-t\right)\left\{\frac{2}{3}-\cos \omega t+\frac{1}{3} \cos ^{3} \omega t\right\}+\frac{1}{3} \sqrt{\frac{8}{\pi}} \theta\left(t-\tau_{c}\right),
$$

and the linear damping function $\gamma_{1}(t)$ can be written as

$$
\zeta_{1}(t)=\zeta_{0}(t)+\lambda^{2} \varepsilon_{1}(t)+N \lambda^{2} \varepsilon_{2}(t) .
$$

The main contribution to the overall damping coefficient $\zeta_{1}(t)$ is given by the function $\zeta_{0}(t)=\omega \int_{0}^{t} d \tau \xi_{0}(\tau)$, while the corrections arising at higher orders in the $\lambda$-expansion are

$$
\begin{aligned}
& \varepsilon_{1}(t)=-\omega \int_{0}^{t} d \tau\left\{2 \xi_{1}(\tau)+\xi_{2}(\tau)\right\} \\
& \varepsilon_{2}(t)=\omega^{3} \int_{0}^{t} d \tau \xi_{0}(\tau) \int_{t-\tau}^{t} d \tau^{\prime} \int_{0}^{\tau^{\prime}} d \tau^{\prime \prime} \xi_{0}\left(\tau^{\prime \prime}\right) .
\end{aligned}
$$

Note that $\varepsilon_{1}(t)$ and $\varepsilon_{2}(t)$ are of different signs for all $t$. If $N \ll 1$ the correction is determined mostly by $\varepsilon_{1}(t)<0$ and tends to decrease the damping function $\zeta_{1}(t)$. In contrast, if $N \gg 1$ the main correction comes from $\varepsilon_{2}(t)>0$ effectively increasing the linear damping.

For a coarse-grained description on the time-scale $t \gg \tau_{c}$ with a time resolution $\tau_{c} \ll \Delta t \ll \tau_{p}$, one can replace the damping functions $\gamma_{i}(t)$ in the LE (81) by their limiting values $\gamma_{i}\left(\tau_{c}\right)$.

\section{CONCLUDING REMARKS}

The notion that the character of Brownian motion of a finite-sized particle may depend on parameters other than the mass ratio $\lambda^{2}$ dates back to Lorentz and has been examined by many authors (see [17-19] and references therein). It is known that when hydrodynamic effects are important another relevant parameter is the ratio of the mass density of the bath to that of the particle. In this paper we have demonstrated that even when hydrodynamic effects are absent, as in the extended Rayleigh model, the character of the behavior of a tagged particle may not governed by $\lambda^{2}$ but by the renormalized parameter $\lambda_{*}^{2}=N \lambda^{2}$, which can be interpreted as the ratio of the average total mass of particles in the interaction zone $M_{*}$ to the mass $M$ of the tagged particle. When the average number of particles in the interaction zone is large (i.e. $N \gg 1$ ), $\lambda_{*} \ll 1$ is a necessary condition for the applicability of a conventional perturbation scheme of derivation of the LE. In this case the conventional assumption of Gaussian random force is justified for any time scale. If $N<1$, the parameter of the expansion is $\lambda^{2}$, and the Gaussian force approximation holds only on a time scale that is much longer than the characteristic time for the relaxation of the bath.

Although this paper focuses on the specific model of an ideal gas bath interacting with a Brownian particle through a quadratic repulsive potential, many of the results obtained are quite general. In particular, the LE (64) and the expressions (66)-(67) for the damping coefficients in terms of microscopic time-correlation functions, which may be considered as the generalized version of the fluctuation-dissipation theorem, are limited neither to the specific form of interaction potential between the bath and the tagged particle nor to the ideal gas bath. The results of section 3 concerning the cumulant expansion of the kernel $K(t)=\left\langle F F^{\dagger}(t)\right\rangle$ are also general and not limited to any specific model. Combined with quite general theorems about cumulant properties [20], these results may be useful for more realistic models with interacting bath particles.

The explicit expression for the kinetic coefficients and memory functions appearing in the Langevin equations have been derived in this paper in the thermodynamic limit, so that any correlations due to finite size of the system are neglected. It should be mentioned, however, that the equations themselves, as well as the fluctuation-dissipation relations relating the kinetic coefficients to correlation functions, also hold for a system with finite baths. For finite systems the explicit form of the kinetic coefficients may be rather complicated even for a bath composed of ideal gas particles.

In this paper we have considered the case of the totally symmetric bath when thermodynamic and microscopic properties of the gas to the left and to the right of the piston are the same. Some interesting physical implications arising in the case of an asymmetric bath will be presented elsewhere [21]. 


\section{ACKNOWLEDGMENTS}

This work was supported by a grant from the Natural Sciences and Engineering Research Council of Canada and funds from the Premier's Research Excellence Award.

\section{APPENDIX A}

In this Appendix, for the sake of completeness, we show explicitly that the function $C_{4}$ defined by Eqs. (14) is of second order in cumulants of products of $G_{i} . C_{4}$ can be written as the sum

$$
C_{4}=C_{4}^{(1)}+C_{4}^{(2)}+C_{4}^{(3)}+C_{4}^{(4)},
$$

where the first constituent,

$$
C_{4}^{(1)}=\frac{2}{m^{2}}\left\{\left\langle G_{0} G_{0}\left(t-t_{1}\right) G_{0}\left(t-t_{2}\right) G_{2}\left(t, t_{3}, t_{4}\right)\right\rangle\right.
$$

$$
\left.-\left\langle G_{0} G_{0}\left(t-t_{1}\right)\right\rangle\left\langle G_{0}\left(t-t_{2}\right) G_{2}\left(t, t_{3}, t_{4}\right)\right\rangle\right\}
$$

is obviously quadratic in cumulants,

$$
\begin{aligned}
C_{4}^{(1)}= & \frac{2}{m^{2}}\left\{\left\langle\left\langle G_{0} G_{0}\left(t-t_{2}\right)\right\rangle\right\rangle\left\langle\left\langle G_{0}\left(t-t_{1}\right) G_{2}\left(t, t_{3}, t_{4}\right)\right\rangle\right\rangle\right. \\
& +\left\langle\left\langle G_{0}\left(t-t_{1}\right) G_{0}\left(t-t_{2}\right)\right\rangle\right\rangle\left\langle\left\langle G_{0} G_{2}\left(t, t_{3}, t_{4}\right)\right\rangle\right\rangle \\
& \left.+\left\langle\left\langle G_{0} G_{0}\left(t-t_{1}\right) G_{0}\left(t-t_{2}\right) G_{2}\left(t, t_{3}, t_{4}\right)\right\rangle\right\rangle\right\} .
\end{aligned}
$$

The second term is

$$
\begin{aligned}
C_{4}^{(2)}= & \frac{1}{m^{2}}\left\{\left\langle G_{0} G_{0}\left(t-t_{1}\right) A_{1}\right\rangle-\left\langle G_{0} G_{0}\left(t-t_{1}\right)\right\rangle\left\langle A_{1}\right\rangle\right. \\
& -\left\langle G_{1}\left(t, t_{4}\right)\right\rangle\left[\left\langle G_{0} G_{0}\left(t-t_{1}\right) G_{1}\left(t-t_{3}, t_{2}-t_{3}\right)\right\rangle\right. \\
& \left.\left.-\left\langle G_{0} G_{0}\left(t-t_{1}\right)\right\rangle\left\langle G_{1}\left(t-t_{3}, t_{2}-t_{3}\right)\right\rangle\right]\right\}
\end{aligned}
$$

where $A_{1}=S\left(t-t_{2}\right) G_{0}\left(t_{2}-t_{3}\right) G_{1}\left(t_{2}, t_{4}\right)$. Noting that $A_{1}$ can be written as

$$
A_{1}=G_{1}\left(t-t_{3}, t_{2}-t_{3}\right) G_{1}\left(t, t_{4}\right)+G_{0}\left(t-t_{3}\right) G_{2}\left(t, t_{2}, t_{4}\right)
$$

and recalling that due to symmetry $\left\langle G_{2}(t)\right\rangle$ and $\left\langle G_{0} G_{1}(t)\right\rangle$ are zero at all times (see Eq. (21)), one can see that $C_{4}^{(2)}$ is also quadratic in cumulants,

$$
\begin{aligned}
C_{4}^{(2)}= & \frac{1}{m^{2}}\left\{\left\langle G_{1}\left(t-t_{3}, t_{2}-t_{3}\right)\right\rangle\left\langle\left\langle G_{0} G_{0}\left(t-t_{1}\right) G_{1}\left(t, t_{4}\right)\right\rangle\right\rangle\right. \\
& +\left\langle\left\langle G_{0} G_{0}\left(t-t_{3}\right)\right\rangle\right\rangle\left\langle\left\langle G_{0}\left(t-t_{1}\right) G_{2}\left(t, t_{2}, t_{4}\right)\right\rangle\right\rangle \\
& +\left\langle\left\langle G_{0} G_{2}\left(t, t_{2}, t_{4}\right)\right\rangle\right\rangle\left\langle\left\langle G_{0}\left(t-t_{1}\right) G_{0}\left(t-t_{3}\right)\right\rangle\right\rangle \\
& +\left\langle\left\langle G_{0} G_{0}\left(t-t_{1}\right) G_{1}\left(t-t_{3}, t_{2}-t_{3}\right) G_{1}\left(t, t_{4}\right)\right\rangle\right\rangle \\
& \left.+\left\langle\left\langle G_{0} G_{0}\left(t-t_{1}\right) G_{0}\left(t-t_{3}\right) G_{2}\left(t, t_{2}, t_{4}\right) G_{1}\left(t, t_{4}\right)\right\rangle\right\rangle\right\} .
\end{aligned}
$$

The third term is

$$
C_{4}^{(3)}=\frac{P_{*}^{2}}{m^{3}}\left\{\left\langle G_{0} B_{1}\right\rangle-\left\langle G_{0} B_{2}\right\rangle\left\langle G_{1}\left(t_{2}, t_{4}\right)\right\rangle+2\left\langle G_{0} B_{3}\right\rangle\right\},
$$

where

$$
\begin{aligned}
& B_{1}=S\left(t-t_{1}\right) S\left(t-t_{2}\right) G_{0}\left(t_{2}-t_{3}\right) G_{1}\left(t_{2}, t_{4}\right) \\
& B_{2}=S\left(t-t_{1}\right) S\left(t-t_{2}\right) G_{0}\left(t_{2}-t_{3}\right) \\
& B_{3}=S\left(t-t_{1}\right) G_{0}\left(t_{1}-t_{2}\right) G_{2}\left(t_{1}, t_{3}, t_{4}\right)
\end{aligned}
$$


can be expressed as

$$
\begin{aligned}
B_{1} & =G_{2}\left(t-t_{3}, t_{1}-t_{3}, t_{2}-t_{3}\right) G_{1}\left(t, t_{4}\right)+G_{1}\left(t-t_{3}, t_{2}-t_{3}\right) G_{2}\left(t, t_{1}, t_{4}\right) \\
& +G_{1}\left(t-t_{3}, t_{1}-t_{3}\right) G_{2}\left(t, t_{2}, t_{4}\right)+G_{0}\left(t-t_{3}\right) G_{3}\left(t, t_{1}, t_{2}, t_{4}\right), \\
B_{2} & =G_{2}\left(t-t_{3}, t_{1}-t_{3}, t_{2}-t_{3}\right), \\
B_{3} & =G_{1}\left(t-t_{2}, t_{1}-t_{2}\right) G_{2}\left(t, t_{3}, t_{4}\right)+G_{0}\left(t-t_{2}\right) G_{3}\left(t, t_{1}, t_{3}, t_{4}\right) .
\end{aligned}
$$

Then using the symmetry property (21) one can see that $C_{4}^{(3)}$ is quadratic in cumulants.

The remaining term

$$
\begin{aligned}
C_{4}^{(4)}= & \left(\frac{P_{*}}{m}\right)^{2}\left\langle G_{0} G_{4}\left(t, t_{1}, t_{2}, t_{3}, t_{4}\right)\right\rangle \\
+ & \frac{3 P_{*}^{2}}{m^{3}}\left\{\left\langle G_{0} G_{0}\left(t-t_{1}\right) G_{3}\left(t, t_{2}, t_{3}, t_{4}\right)\right\rangle\right. \\
& \left.-\left\langle G_{0} G_{0}\left(t-t_{1}\right)\right\rangle\left\langle G_{3}\left(t, t_{2}, t_{3}, t_{4}\right)\right\rangle\right\}
\end{aligned}
$$

is clearly linear in cumulants,

$$
\begin{aligned}
C_{4}^{(4)}= & \left(\frac{P_{*}}{m}\right)^{2}\left\langle\left\langle G_{0} G_{4}\left(t, t_{1}, t_{2}, t_{3}, t_{4}\right)\right\rangle\right\rangle \\
& +\frac{3 P_{*}^{2}}{m^{3}}\left\langle\left\langle G_{0} G_{0}\left(t-t_{1}\right) G_{3}\left(t, t_{2}, t_{3}, t_{4}\right)\right\rangle\right\rangle .
\end{aligned}
$$

\section{APPENDIX B}

In this Appendix the functions $G_{i}$ defined by Eqs. (19) are evaluated for the extended Rayleigh model of diffusion. These functions are defined in terms of powers of alternating operators $\partial / \partial X$ and $e^{\mathcal{L}_{0}\left(t_{i}-t_{k}\right)}$. As mentioned in the text, the representation (33), (35) for the force on the fixed piston is unwieldy since it involves $q_{i}(t)$ and $v_{i}(t)$ which are functions of $X$. For the purpose of evaluating the $G_{i}$, it is convenient to express the force in terms of $N(x, v) \equiv N(x, v ; t=0)$. For $t>0$, we have

$$
\begin{aligned}
F_{l}(t)= & k_{f} \int_{0}^{\infty} d v \int_{-v t}^{a(t)} d q N\left(X_{l}+q, v\right) \frac{v}{\omega} \sin \omega\left(t+\frac{q}{v}\right) \\
& +\theta\left(\tau_{c} / 2-t\right) k_{f} \int_{0}^{\infty} d v \int_{0}^{\infty} d q N\left(X_{l}+q, v\right) q(t) \\
& +\theta\left(\tau_{c} / 2-t\right) k_{f} \int_{-\infty}^{0} d v \int_{Q(t)}^{\infty} d q N\left(X_{l}+q, v\right) q(t) \\
& +\theta\left(t-\tau_{c} / 2\right) \theta\left(\tau_{c}-t\right) k_{f} \int_{0}^{\infty} d v \int_{0}^{Q(t)} d q N\left(X_{l}+q, v\right) q(t),
\end{aligned}
$$

where

$$
\begin{aligned}
a(t) & =-v \theta\left(t-\tau_{c}\right)\left(t-\tau_{c}\right), \\
q(t) & =q \cos \omega t+\frac{v}{\omega} \sin \omega t \\
Q(t) & =-\frac{v}{\omega} \tan \omega t
\end{aligned}
$$

The first term in Eq. (B1) describes the contribution to the force $F_{l}(t)$ from the particles that were outside the interaction zone at $t=0$ (i.e. $q<0$ ) and are in the interaction zone at the moment $t$ (i.e. $q(t)>0$ ). 
The remaining terms give the contribution from particles that were in the interaction zone at $t=0$ (i.e. $q>0$ ) and are still there at the moment $t$ (i.e. $q(t)>0$ ). In fact, all particles in the interaction zone with positive initial velocities at $t=0$ will be still in the interaction zone at $t<\tau_{c} / 2$ (the second term), while the particles with negative initial velocities will be in the interaction zone at time $t<\tau_{c} / 2$ only if at $t=0$ they reside deep inside the interaction zone, namely $q>Q(t)$ (the third term). For $\tau_{c}>t>\tau_{c} / 2$ only the particles with positive initial velocity will be in the interaction zone at time $t$ provided their initial coordinates are less than $Q(t)$ (the last term).

From expression (B1), one easily calculates $\partial F_{l}(t) / \partial X$ writing $\partial N\left(X_{l}+q, v\right) / \partial X=\partial N\left(X_{l}+q\right) / \partial q$ and integrating by parts to obtain,

$$
\begin{aligned}
\frac{\partial F_{l}(t)}{\partial X}= & -k_{f} \cos \omega t N_{z}(t) \\
& -k_{f} \int_{0}^{\infty} d v \int_{-v t}^{a(t)} d q N\left(X_{l}+q, v\right) \frac{v}{\omega} \cos \omega\left(t+\frac{q}{v}\right) .
\end{aligned}
$$

Here $N_{z}(t)$ is the number of particles which were in the interaction zone at $t=0$ and remain at $t>0$,

$$
\begin{aligned}
N_{z}(t)= & \theta\left(\tau_{c} / 2-t\right) \int_{0}^{\infty} d v \int_{0}^{\infty} d q N\left(X_{l}+q, v\right) \\
& +\theta\left(\tau_{c} / 2-t\right) \int_{-\infty}^{0} d v \int_{Q(t)}^{\infty} d q N\left(X_{l}+q, v\right) \\
& +\theta\left(t-\tau_{c} / 2\right) \theta\left(\tau_{c}-t\right) \int_{0}^{\infty} d v \int_{0}^{Q(t)} d q N\left(X_{l}+q, v\right) .
\end{aligned}
$$

$N_{z}(t)$ can be written in compact form in terms of the density at time $-\tau_{c}$ according to,

$$
N_{z}(t)=\theta\left(\tau_{c}-t\right) \int_{0}^{\infty} d v \int_{-v \tau_{c}}^{-v t} d q N\left(X_{l}+q, v ;-\tau_{c}\right) .
$$

In fact, the number of particles in the interaction zone at $t=0$ is

$$
\int_{0}^{\infty} d v \int_{-v \tau_{c}}^{0} d q N\left(X_{l}+q, v ;-\tau_{c}\right)
$$

while at time $t$ it is given by

$$
\int_{0}^{\infty} d v \int_{-v \tau_{c}}^{0} d q N\left(X_{l}+q, v ; t-\tau_{c}\right)
$$

$$
=\int_{0}^{\infty} d v \int_{-v\left(t+\tau_{c}\right)}^{-v t} d q N\left(X_{l}+q, v ;-\tau_{c}\right) .
$$

By definition $N_{z}(t)$ involves the particles which contribute to both integrals (B5) and (B6), which leads to Eq. (B4). Combining Eqs. (B2) and (B4), we have

$$
\begin{aligned}
\frac{\partial F_{l}(t)}{\partial X}= & -k_{f} \int_{0}^{\infty} d v \int_{-v t}^{a(t)} d q N\left(X_{l}+q, v\right) \cos \omega\left(t+\frac{q}{v}\right) \\
& -k_{f} \theta\left(\tau_{c}-t\right) \cos \omega t \int_{0}^{\infty} d v \int_{-v \tau_{c}}^{-v t} d q N\left(X_{l}+q, v ;-\tau_{c}\right) .
\end{aligned}
$$


For comparison, let us calculate the average derivative of the total force $F_{0}(t)=F_{l}+F_{r}$,

$$
\begin{aligned}
\left\langle\frac{\partial F_{0}(t)}{\partial X}\right\rangle=2\left\langle\frac{\partial F_{l}(t)}{\partial X}\right\rangle & =-\frac{2 k_{f} n S}{\omega} \int_{0}^{\infty} f_{M}(v) v d v \theta\left(\tau_{c}-t\right)(\sin \omega t+(\pi-\omega t) \cos \omega t)
\end{aligned}
$$

Comparing this expression with Eq. (43) for the memory kernel $K_{0}(t)=\left\langle F F_{0}(t)\right\rangle$, it is evident that $\left\langle\partial F_{0}(t) / \partial X\right\rangle=$ $-\beta\left\langle F F_{0}(t)\right\rangle$. This is the general result used in the main text, Eq. (10) confirmed using the explicit expression for $\partial F_{0}(t) / \partial X$.

¿From Eq. (B7), we obtain the following expression for $G_{l 1}$ :

$$
\begin{aligned}
& G_{l 1}\left(t_{1}, t_{2}\right) \equiv e^{\mathcal{L}_{0}\left(t_{1}-t_{2}\right)} \frac{\partial F_{l}\left(t_{2}\right)}{\partial X} \\
& =-k_{f} \int_{0}^{\infty} d v \int_{-v t_{2}}^{a\left(t_{2}\right)} d q N\left(X_{l}+q, v ; t_{1}-t_{2}\right) \cos \omega\left(t_{2}+\frac{q}{v}\right) \\
& -k_{f} \theta\left(\tau_{c}-t_{2}\right) \cos \omega t_{2} \int_{0}^{\infty} d v \int_{-v \tau_{c}}^{-v t_{2}} d q N\left(X_{l}+q, v ; t_{1}-t_{2}-\tau_{c}\right)
\end{aligned}
$$

where, according to (13), it is assumed that $t_{1}>t_{2}$. Expressing this in terms of the microscopic density at time $-\tau_{c}$ using (37), one obtains Eq. (68) of the main text.

To evaluate $G_{2}$, one has take the derivative of $G_{1}$ with respect to $X$. Let us express $G_{1}$ in terms of the density at time $t=0, N(x, v)$, as done above for $F_{l}(t)$. The first term in the right-hand side of Eq. (B8) involves only particles located outside the interaction zone, so using the property (37), the first term can be written as

$$
\begin{aligned}
-k_{f} \int_{0}^{\infty} d v \int_{-v t_{2}}^{a\left(t_{2}\right)} d q N\left(X_{l}+q-v\left(t_{1}-t_{2}\right), v\right) \cos \omega\left(t_{2}+\frac{q}{v}\right) & \\
& =-k_{f} \int_{0}^{\infty} d v \int_{-v t_{1}}^{b} d q N\left(X_{l}+q, v\right) \cos \omega\left(t_{1}+\frac{q}{v}\right)
\end{aligned}
$$

where the upper integration limit is

$$
b=-v\left(t_{1}-t_{2}\right) \theta\left(\tau_{c}-t_{2}\right)-v\left(t_{1}-\tau_{c}\right) \theta\left(t_{2}-\tau_{c}\right) .
$$

The second term on the right-hand side of Eq. (B8) can be expressed as

$$
\begin{aligned}
-k_{f} \theta\left(\tau_{c}-t_{2}\right) \cos \omega t_{2} \int_{0}^{\infty} d v \int_{-v \tau_{c}}^{-v t_{2}} d q N\left(X_{l}+q-v\left(t_{1}-t_{2}\right), v ;-\tau_{c}\right) & \\
& =-k_{f} \theta\left(\tau_{c}-t_{2}\right) \cos \omega t_{2} \int_{0}^{\infty} d v \int_{-v\left(\tau_{c}+t_{1}-t_{2}\right)}^{-v t_{1}} d q N\left(X_{l}+q, v ;-\tau_{c}\right) .
\end{aligned}
$$

If $t_{1}>\tau_{c}$, then all particles which contribute this integral at $t=0$ will be outside the interaction zone in the $q$-interval from $-v\left(t_{1}-t_{2}\right)$ to $-v\left(t_{1}-\tau_{c}\right)$. Therefore, for $t_{1}>\tau_{c}$ the second term equals

$$
-k_{f} \theta\left(\tau_{c}-t_{2}\right) \theta\left(t_{1}-\tau_{c}\right) \cos \omega t_{2} \int_{0}^{\infty} d v \int_{-v\left(t_{1}-t_{2}\right)}^{-v\left(t_{1}-\tau_{c}\right)} d q N\left(X_{l}+q, v\right) .
$$


If $t_{1}<\tau_{c}$, then two sets of particles contribute to expression (B10). The first set of particles is composed of particles which at time $-\tau_{c}$ are in the $q$-interval from $-v\left(\tau_{c}+t_{1}-t_{2}\right)$ to $-v \tau_{c}$. At $t=0$, these particles will be outside the interaction zone in the interval $\left(-v\left(t_{1}-t_{2}\right), 0\right)$, and hence their contribution is

$$
-k_{f} \cos \omega t_{2} \int_{0}^{\infty} d v \int_{-v\left(t_{1}-t_{2}\right)}^{0} d q N\left(X_{l}+q, v\right) .
$$

The second group of particles are those that were in the $q$-interval from $-v \tau_{c}$ to $-v t_{1}$ at time $-\tau_{c}$. At $t=0$, all these particles will be in the interaction zone. Taking into account Eq. (B4), the corresponding contribution can be written as $-k_{f} \cos \omega t_{2} N_{z}\left(t_{1}\right)$. Using expression (B3) for $N_{z}(t)$, one arrives at the following representation for $G_{l 1}$ in terms of $N(x, v)$,

$$
\begin{aligned}
G_{l 1}\left(t_{1}, t_{2}\right)= & -k_{f} \int_{0}^{\infty} d v \int_{-v t_{1}}^{b} d q N\left(X_{l}+q, v\right) \cos \omega\left(t_{1}+\frac{q}{v}\right) \\
& -k_{f} \theta\left(\tau_{c}-t_{2}\right) \theta\left(t_{1}-\tau_{c}\right) \cos \omega t_{2} \int_{0}^{\infty} d v \int_{-v\left(t_{1}-t_{2}\right)}^{-v\left(t_{1}-\tau_{c}\right)} d q N\left(X_{l}+q, v\right) \\
& -k_{f} \theta\left(\tau_{c}-t_{2}\right) \theta\left(\tau_{c}-t_{1}\right) \cos \omega t_{2} \int_{0}^{\infty} d v \int_{-v\left(t_{1}-t_{2}\right)}^{0} d q N\left(X_{l}+q, v\right) \\
& -k_{f} \theta\left(\tau_{c}-t_{2}\right) \theta\left(\tau_{c} / 2-t_{1}\right) \cos \omega t_{2} \int_{0}^{\infty} d v \int_{0}^{\infty} d q N\left(X_{l}+q, v\right) \\
& -k_{f} \theta\left(\tau_{c}-t_{2}\right) \theta\left(\tau_{c} / 2-t_{1}\right) \cos \omega t_{2} \int_{-\infty}^{0} d v \int_{Q\left(t_{1}\right)}^{\infty} d q N\left(X_{l}+q, v\right) \\
& -k_{f} \theta\left(\tau_{c}-t_{2}\right) \theta\left(\tau_{c}-t_{1}\right) \theta\left(t_{1}-\tau_{c} / 2\right) \cos \omega t_{2} \times \\
& \int_{0}^{\infty} d v \int_{0}^{Q\left(t_{1}\right)} d q N\left(X_{l}+q, v\right) .
\end{aligned}
$$

Taking derivative of this expression with respect to $X$ gives

$$
\begin{aligned}
\frac{\partial}{\partial X} G_{l 1}\left(t_{1}, t_{2}\right)= & k_{f} \int_{0}^{\infty} d v N\left(X_{l}-v t_{1}, v\right) \\
& +k_{f}\left[\theta\left(t_{2}-\tau_{c}\right)-\theta\left(\tau_{c}-t_{2}\right) \theta\left(t_{1}-\tau_{c}\right) \cos \omega t_{2}\right] \int_{0}^{\infty} d v N\left(X_{l}-v\left(t_{1}-\tau_{c}\right), v\right) \\
& -k_{f} \int_{0}^{\infty} d v \int_{-v t_{1}}^{b} d q N\left(X_{l}+q, v\right) \frac{\omega}{v} \sin \omega\left(t_{1}+\frac{q}{v}\right) \\
& +\theta\left(\tau_{c} / 2-t_{1}\right) \theta\left(\tau_{c}-t_{2}\right) k \cos \omega t_{2} \int_{-\infty}^{0} d v N\left(X_{l}+Q\left(t_{1}\right), v\right) \\
& -\theta\left(\tau_{c}-t_{1}\right) \theta\left(t_{1}-\tau_{c} / 2\right) \theta\left(\tau_{c}-t_{2}\right) k \cos \omega t_{2} \int_{0}^{\infty} d v N\left(X_{l}+Q\left(t_{1}\right), v\right) .
\end{aligned}
$$


The last two terms can be written as

$$
\begin{aligned}
& k_{f} \theta\left(\tau_{c}-t_{2}\right) \theta\left(\tau_{c}-t_{1}\right) \frac{\cos \omega t_{2}}{\tan \omega t_{1}} \int_{0}^{\infty} d v N\left(X_{l}+\frac{v}{\omega},-\frac{v}{\tan \omega t_{1}}\right) \\
&=k_{f} \theta\left(\tau_{c}-t_{2}\right) \theta\left(\tau_{c}-t_{1}\right) \frac{\cos \omega t_{2}}{\tan \omega t_{1}} \int_{0}^{\infty} d v N\left(X_{l}-\frac{v t_{1}}{\sin \omega t_{1}}, \frac{v}{\sin \omega t_{1}} ;-\tau_{c}\right) \\
&=k_{f} \theta\left(\tau_{c}-t_{2}\right) \theta\left(\tau_{c}-t_{1}\right) \cos \omega t_{1} \cos \omega t_{2} \int_{0}^{\infty} d v N\left(X_{l}-v t_{1}, v ;-\tau_{c}\right),
\end{aligned}
$$

where we have used the property that if the initial coordinate and velocity of the particle in the interaction zone are

$$
q(0)=\frac{V}{\omega}, \quad v(0)=-\frac{V}{\tan \omega t}
$$

with $V>0$ and $0<t<\tau_{c}$, then at $t=-\tau_{c}$

$$
q\left(-\tau_{c}\right)=-v\left(-\tau_{c}\right) t, \quad v\left(-\tau_{c}\right)=\frac{V}{\sin \omega t} .
$$

Substituting (B15) into (B15), acting on the result by the propagator $e^{\mathcal{L}_{0}\left(t-t_{1}\right)}$, and using again the property $N(x, v ; t+\tau)=N(x-v \tau, v ; t)$ for the motion outside the interaction zone, we finally obtain for $t>t_{1}>t_{2}$,

$$
\begin{aligned}
G_{l 2}\left(t, t_{1}, t_{2}\right) \equiv & e^{\mathcal{L}_{0}\left(t-t_{1}\right)} \frac{\partial}{\partial X} G_{1}\left(t_{1}, t_{2}\right) \\
= & k_{f} \int_{0}^{\infty} d v N\left(X_{l}-v t, v\right) \\
& +k_{f} \theta\left(t_{1}-\tau_{c}\right) \theta\left(t_{2}-\tau_{c}\right) \int_{0}^{\infty} d v N\left(X_{l}-v\left(t-\tau_{c}\right), v\right) \\
& -k_{f} \theta\left(\tau_{c}-t_{2}\right) \theta\left(t_{1}-\tau_{c}\right) \cos \omega t_{2} \int_{0}^{\infty} d v N\left(X_{l}-v\left(t-\tau_{c}\right), v\right) \\
& -k_{f} \int_{0}^{\infty} d v \int_{-v t}^{b} d q N\left(X_{l}+q, v\right) \frac{\omega}{v} \sin \omega\left(t+\frac{q}{v}\right) \\
& +k_{f} \theta\left(\tau_{c}-t_{2}\right) \theta\left(\tau_{c}-t_{1}\right) \cos \omega t_{1} \cos \omega t_{2} \int_{0}^{\infty} d v N\left(X_{l}-v t, v ;-\tau_{c}\right)
\end{aligned}
$$

where $b=-v\left(t-t_{2}\right) \theta\left(\tau_{c}-t_{2}\right)-v\left(t-\tau_{c}\right) \theta\left(t_{2}-\tau_{c}\right)$. Expressing the first three terms through $N\left(x, v ;-\tau_{c}\right)$ rather than $N(x, v)$, one arrives at Eq. (71) of the main text.

[1] R. Zwanzig, Noneqiuilibrium Statistical Mechanics, Oxford University Press, 2001.

[2] N. G. van Kampen, Stochastic Processes in Physics and Chemistry, (North Holland, Amsterdam, 1992).

[3] P. Mazur and D. Bedeaux, Physics A 174, 155 (1991); P. Mazur, Phys. Rev. A 45, 8957 (1992); J. B. Avalos and I. Pagonabarraga, Phys. Rev. E 52, 5881 (1995).

[4] J. T. Hynes, R. Kapral, and M. Weinberg, Physics A 80, 105 (1975).

[5] P. Mazur and I. Oppenheim, Physics A 50, 241 (1970). 
[6] J. M. Deutch and R. Silbey, Phys. Rev. A 3, 2049 (1971); T. Morita and H. Mori, Prog. Theor. Phys. 56, 498 (1976).

[7] Note however that the Langevin equation for powers of the momentum for a tagged particle in a harmonic chain are less specific and do exibit many features characteristic for realistic systems, see J. T. Hynes, R. Kapral, and M. Weinberg, J. Stat. Phys. 13, 427 (1975).

[8] Lord Rayleigh, Phil. Mag. 32, 424 (1891).

[9] J. L. Lebowitz, Phys. Rev. 114, 1192 (1959).

[10] N. G. van Kampen, Can. J. Phys. 39, 551 (1961); C.T.J. Alkemade, N.G.van Kampen, and D.K.C. MacDonald, Proc. R. Soc. 271 A, 449 (1963).

[11] J. T. Hynes, J. Chem. Phys. 62, 2972 (1975).

[12] E. Lieb, Physica A 263, 491 (1999); Ch. Gruber and J. Piasecki, ibid 268, 412 (1999); E. Kestemont, C.Van den Broeck, and M. M. Mansour, Europhys. Lett 49, 143 (2000); T. Munakata and H. Ogawa, Phys. Rev. E 64, 036119 (2001); Ch. Gruber, S. Pache, and A. Lesne, J. Stat. Phys. 108, 669 (2002); J. Piasecki, J. Phys.: Condens. Matter 14, 9265 (2002); N. Chernov and J. L. Lebowitz, ibid. 109, 507 (2002); N. Chernov, J. L. Lebowitz and Ya. Sinai, ibid 109, 529 (2002).

[13] A simple way to prove this formula is to integrate the identity $\frac{d}{d t} e^{\mathcal{A} t} e^{-(\mathcal{A}+\mathcal{B}) t}=-e^{\mathcal{A} t} \mathcal{B} e^{-(\mathcal{A}+\mathcal{B}) t}$.

[14] We have used the identity $f(t-\tau)=f(t)-\int_{t-\tau}^{t} d \tau^{\prime} \dot{f}\left(\tau^{\prime}\right)$.

[15] J. T. Hynes, R. Kapral, and M. Weinberg, Physica A 81, 485 (1975); H. Mori, H. Fujisaka and H. Shigematsu, Prog. Theor. Phys. 51, 109 (1974).

[16] D. K. C. MacDonald, Phys. Rev. 108, 541 (1957).

[17] M. Tokuyama and I. Oppenheim, Physica A 94, 501 (1978).

[18] J. N. Roux, ibid 188, 526 (1992).

[19] L. Bocquet and J. Piasecki, J. Stat. Phys. 87, 1005 (1997).

[20] J. Schofield and I. Oppenheim, Physica A 187, 210 (1992).

[21] A. V. Plyukhin and J. Schofield, submitted 
FIG. 1. The function $\xi_{0}(t)$ (solid line) governing the time dependence of the memory function $M_{0}(t)=N \omega^{2} \xi_{0}(t)$ in the non-Markovian Langevin equation (15), and the damping function $\zeta_{0}(t)$ (dashed line) in the local Langevin equation (48). 


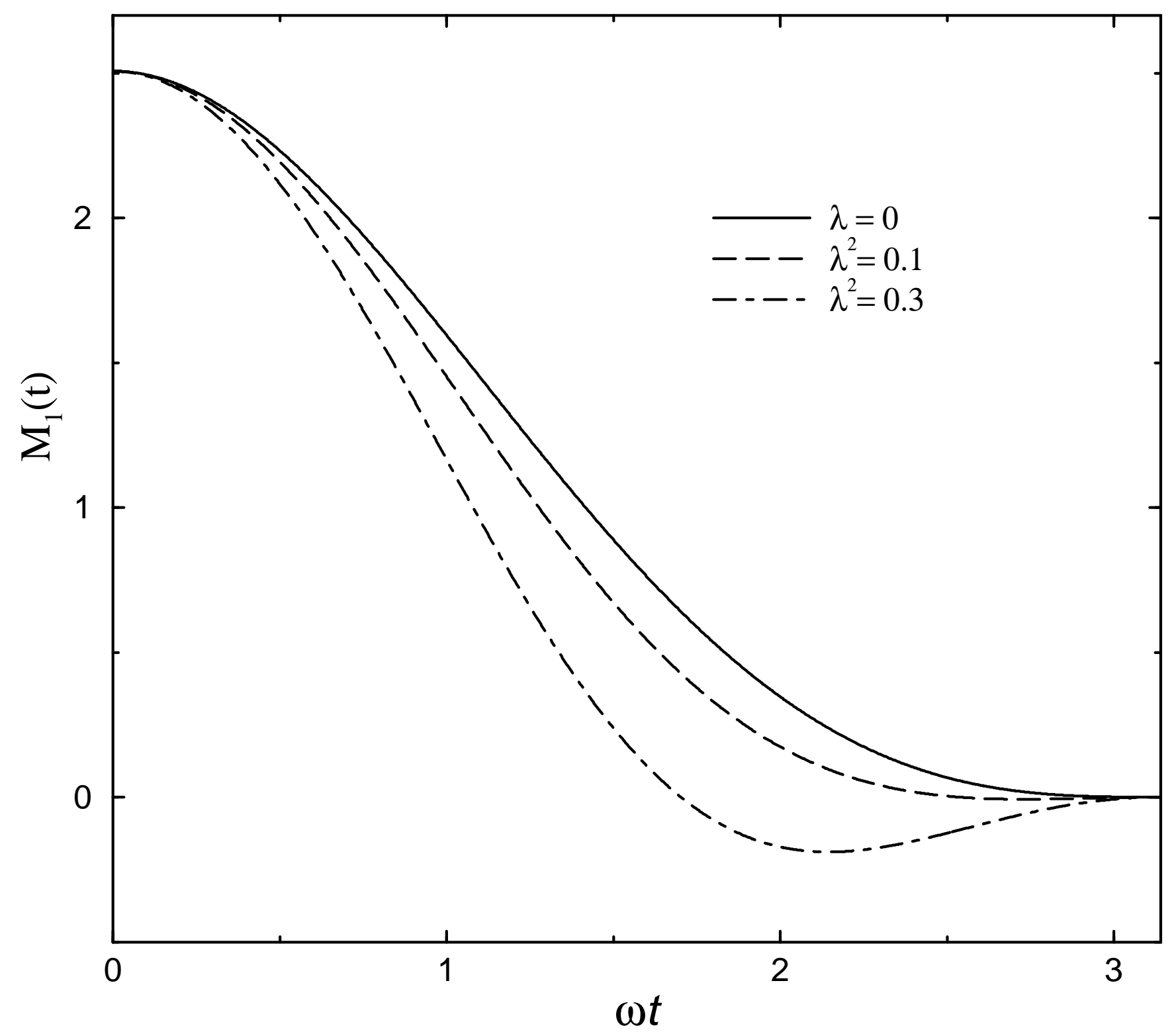

